\title{
Correction of a pathogenic gene mutation in human embryos
}

Hong $\mathrm{Ma}^{1 *}$, Nuria Marti-Gutierrez ${ }^{1 *}$, Sang-Wook Park ${ }^{2 *}$, Jun $\mathrm{Wu}^{3 *}$, Yeonmi Lee ${ }^{1}$, Keiichiro Suzuki ${ }^{3}$, Amy Koski ${ }^{1}$, Dongmei Ji ${ }^{1}$, Tomonari Hayama ${ }^{1}$, Riffat Ahmed ${ }^{1}$, Hayley Darby ${ }^{1}$, Crystal Van Dyken ${ }^{1}$, Ying Li ${ }^{1}$, Eunju Kang ${ }^{1}$, A. - Reum Park ${ }^{2}$, Daesik Kim ${ }^{4}$, Sang-Tae Kim², Jianhui Gong $5,6,7,8$, Ying Gu ${ }^{5,6,7}$, Xun Xu ${ }^{5,6,7}$, David Battaglia ${ }^{1,9}$, Sacha A. Krieg, ${ }^{9}$ David M. Lee ${ }^{9}$, Diana H. Wu ${ }^{9}$, Don P. Wolf ${ }^{1}$, Stephen B. Heitner ${ }^{10}$, Juan Carlos Izpisua Belmonte ${ }^{3}$, Paula Amato $^{1,9} \S$, Jin-Soo Kim ${ }^{2,4} \S$, Sanjiv Kaul ${ }^{10} \S \&$ Shoukhrat Mitalipov ${ }^{1,10} \S$

\begin{abstract}
Genome editing has potential for the targeted correction of germline mutations. Here we describe the correction of the heterozygous MYBPC3 mutation in human preimplantation embryos with precise CRISPR-Cas9-based targeting accuracy and high homology-directed repair efficiency by activating an endogenous, germline-specific DNA repair response. Induced double-strand breaks (DSBs) at the mutant paternal allele were predominantly repaired using the homologous wild-type maternal gene instead of a synthetic DNA template. By modulating the cell cycle stage at which the DSB was induced, we were able to avoid mosaicism in cleaving embryos and achieve a high yield of homozygous embryos carrying the wild-type MYBPC3 gene without evidence of off-target mutations. The efficiency, accuracy and safety of the approach presented suggest that it has potential to be used for the correction of heritable mutations in human embryos by complementing preimplantation genetic diagnosis. However, much remains to be considered before clinical applications, including the reproducibility of the technique with other heterozygous mutations.
\end{abstract}

More than 10,000 monogenic inherited disorders have been identified, affecting millions of people worldwide. Among these are autosomal dominant mutations, where inheritance of a single copy of a defective gene can result in clinical symptoms. Genes in which dominant mutations manifest as late-onset adult disorders include BRCA1 and $B R C A 2$, which are associated with a high risk of breast and ovarian cancers $^{1}$, and $M Y B P C 3$, mutation of which causes hypertrophic cardiomyopathy $(\mathrm{HCM})^{2}$. Because of their delayed manifestation, these mutations escape natural selection and are often transmitted to the next generation. Consequently, the frequency of some of these founder mutations in particular human populations is very high. For example, the MYBPC3 mutation is found at frequencies ranging from $2 \%$ to $8 \%{ }^{3}$ in major Indian populations, and the estimated frequency of both $B R C A 1$ and BRCA2 mutations among Ashkenazi Jews exceeds $2 \%{ }^{4}$.

$\mathrm{HCM}$ is a myocardial disease characterized by left ventricular hypertrophy, myofibrillar disarray and myocardial stiffness; it has an estimated prevalence of 1:500 in adults ${ }^{5}$ and manifests clinically with heart failure. HCM is the commonest cause of sudden death in otherwise healthy young athletes. HCM, while not a uniformly fatal condition, has a tremendous impact on the lives of individuals, including physiological (heart failure and arrhythmias), psychological (limited activity and fear of sudden death), and genealogical concerns. MYBPC3 mutations account for approximately $40 \%$ of all genetic defects causing HCM and are also responsible for a large fraction of other inherited cardiomyopathies, including dilated cardiomyopathy and left ventricular non-compaction ${ }^{6}$. MYBPC3 encodes the thick filament-associated cardiac myosin-binding protein $\mathrm{C}$ (cMyBP-C), a signalling node in cardiac myocytes that contributes to the maintenance of sarcomeric structure and regulation of both contraction and relaxation ${ }^{2}$.

Current treatment options for HCM provide mostly symptomatic relief without addressing the genetic cause of the disease. Thus, the development of novel strategies to prevent germline transmission of founder mutations is desirable. One approach for preventing second-generation transmission is preimplantation genetic diagnosis (PGD) followed by selection of non-mutant embryos for transfer in the context of an in vitro fertilization (IVF) cycle. When only one parent carries a heterozygous mutation, $50 \%$ of the embryos should be mutationfree and available for transfer, while the remaining carrier embryos are discarded. Gene correction would rescue mutant embryos, increase the number of embryos available for transfer and ultimately improve pregnancy rates.

Recent developments in precise genome-editing techniques and their successful applications in animal models have provided an option for correcting human germline mutations. In particular, CRISPR-Cas9 is a versatile tool for recognizing specific genomic sequences and inducing $\mathrm{DSBs}^{7-10}$. DSBs are then resolved by endogenous DNA repair mechanisms, preferentially using a non-homologous end-joining (NHEJ) pathway. Obviously, NHEJ is inappropriate for gene correction applications because it introduces additional mutations in the form of insertions or deletions at the DSB site, commonly referred to as indels. In some cases, however, targeted cells activate an alternative DNA repair pathway called homology-directed repair (HDR) that rebuilds the DSB site using the non-mutant homologous chromosome or a supplied exogenous DNA molecule as a template, leading to correction of the

\footnotetext{
${ }^{1}$ Center for Embryonic Cell and Gene Therapy, Oregon Health \& Science University, 3303 Southwest, Bond Avenue, Portland, Oregon 97239 , USA. ${ }^{2}$ Center for Genome Engineering, Institute for

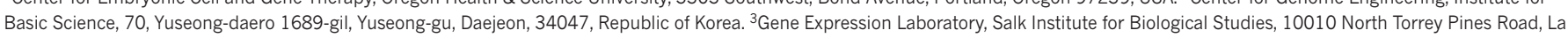
Jolla, California 92037, USA. ${ }^{4}$ Department of Chemistry, Seoul National University, 599 Gwanak-ro, Gwanak-gu, Seoul, 151-747, Republic of Korea. ${ }^{5}$ BGI-Shenzhen, Build 11, Beishan Industrial Zone, Yantian District, Shenzhen, 518083, China. ${ }^{6}$ China National GeneBank, BGI-Shenzhen, Jinsha Road, Dapeng District, Shenzhen, 518210, China. ${ }^{7}$ BGI-Qingdao, 2877 Tuanjie Road, Sino-

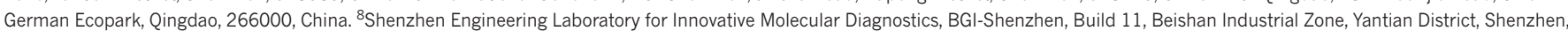
518083, China. ${ }^{9}$ Division of Reproductive Endocrinology and Infertility, Department of Obstetrics and Gynecology, Oregon Health \& Science University, 3303 Southwest, Bond Avenue, Portland, Oregon 97239, USA. ${ }^{10}$ Knight Cardiovascular Institute, Oregon Health \& Science University, 3181 Southwest, Sam Jackson Park Road, Portland, Oregon 97239, USA.

*These authors contributed equally to this work.

$\S$ These authors jointly supervised this work.
} 
mutant allele ${ }^{11,12}$. At present, CRISPR-Cas9 is predominantly used to introduce mutations and in the generation of gene knockouts using intrinsic NHEJ. Because HDR efficiency is relatively low ${ }^{8}$, applications of genome editing for gene therapy have been limited.

In early attempts, the introduction of genome editing constructs into one-cell embryos (zygotes), resulted in multicellular embryos or offspring with mosaic outcomes in individual cells ${ }^{13,14}$. Also, off-target mutations that could be introduced into the developing embryo remained an undesirable possibility.

We sought to investigate human gamete and embryo DNA repair mechanisms activated in response to CRISPR-Cas9-induced DSBs. In an effort to demonstrate the proof-of-principle that heterozygous gene mutations can be corrected in human gametes or early embryos, we focused on the MYBPC3 mutation that has been implicated in HCM. Although homozygous mutations with no PGD alternative would have been most desirable for gene correction, generating homozygous human embryos for research purposes is practically impossible. Homozygous MYBPC3 mutations in adults are extremely rare owing to the severity of the clinical symptoms and early onset of the disease. Therefore, we specifically targeted the heterozygous four-base-pair (bp) deletion in the MYBPC3 gene in human zygotes introduced by heterozygous, carrier sperm while oocytes obtained from healthy donors provided the wild-type allele. By accurate analysis of cleaving embryos at the single-cell level, we show high targeting efficiency and specificity of preselected CRISPR-Cas9 constructs. Moreover, DSBs in the mutant paternal $M Y B P C 3$ gene were preferentially repaired using the wild-type oocyte allele as a template, suggesting an alternative, germlinespecific DNA repair response. Mechanisms responsible for mosaicism in embryos were also investigated and a proposed solution to minimize their occurrence developed-namely the co-injection of sperm and CRISPR-Cas9 components into metaphase II (MII) oocytes.

\section{Subject with a heterozygous MYBPC $3^{\triangle G A G T}$ deletion}

An adult male patient with well-documented familial HCM caused by a heterozygous dominant 4-bp GAGT deletion (g.9836_9839 del., NC_000011.10) in exon 16 of MYBPC3, currently managed with an implantable cardioverter defibrillator and antiarrhythmic medications, agreed to donate skin, blood and semen samples. Skin fibroblast cultures were expanded and used to generate heterozygous patient induced pluripotent stem cells (iPSCs) as described previously ${ }^{15}$. Two single-guide RNA (sgRNA)-Cas9 ${ }^{16-18}$ constructs were designed to target this specific MYBPC $3^{\triangle G A G T}$ deletion (Extended Data Fig. 1a, b) along with two exogenous single-stranded oligodeoxynucleotide (ssODN) templates encoding homology arms to the targeted region (Extended Data Table 1). To differentiate from the wild-type allele, two synonymous singlenucleotide substitutions were introduced into each ssODN template. In addition, ssODN-2 nucleotide substitutions provided an additional restriction enzyme (BstBI) recognition site (Extended Data Fig. 1a, b).

The efficacy and specificity of each construct were tested by transfecting patient iPSCs. Cells were electroporated together with ssODN, Cas 9 and sgRNA expression plasmids and subcloned, and the targeted region for each clone was analysed by sequencing (Extended Data Fig. 1c). Of 61 iPSC clones transfected with CRISPR-Cas9-1, 44 (72.1\%) were not targeted, as evidenced by the presence of both intact wild-type and intact mutant alleles. Among targeted clones, 10 of 17 (58.8\%) were repaired by NHEJ and contained various indels adjacent to the mutation site (Extended Data Fig. 1d, e and Supplementary Table 1). The remaining seven clones were repaired by HDR using ssODN-1 as judged by the presence of the marker nucleotide substitutions. Thus, the total targeting efficiency for CRISPR-Cas9-1 was 27.9\% (17/61). Among the targeted clones, only 41.2\% (7/17) were repaired by HDR (Extended Data Fig. 1e). The targeting efficiency with CRISPRCas9-2 was $13.1 \%(23 / 175)$ and the HDR was considerably lower at $13 \%(3 / 23)$. Of note, among the three HDR-repaired iPSC clones, two were repaired using the ssODN-2 template while the third clone contained intact wild-type sequences in both alleles (Extended Data

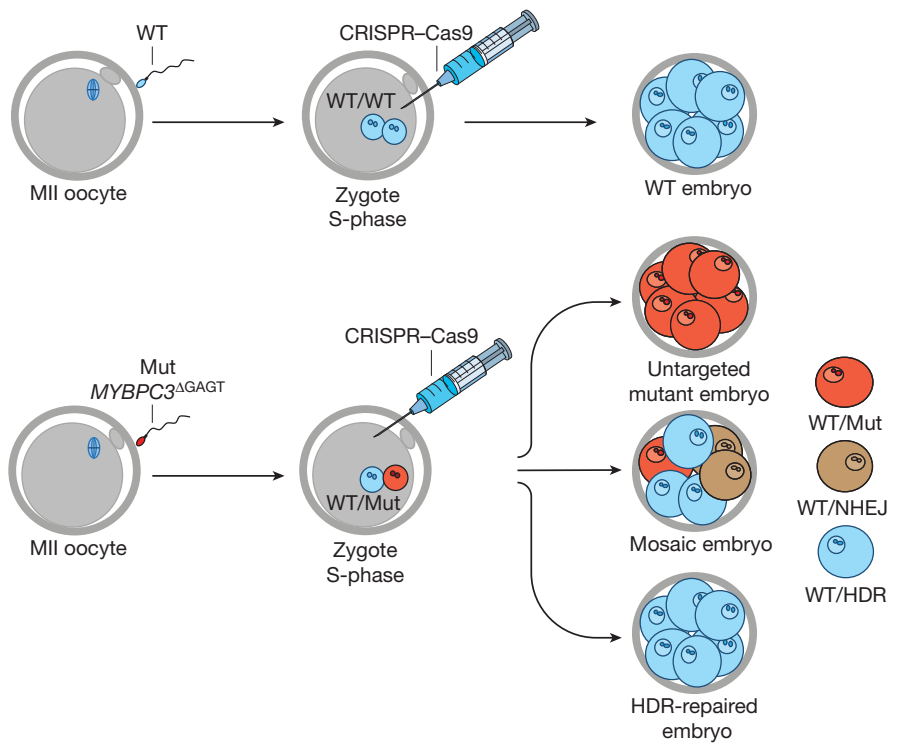

Figure 1 Gene correction in S-phase-injected human embryos. Schematic of $M Y B P C 3^{\triangle \mathrm{GAGT}}$ gene targeting by injection of CRISPR-Cas9 into human zygotes at the S-phase of the cell cycle. MII oocytes were fertilized by sperm from a heterozygous patient with equal numbers of mutant and wild-type (WT) spermatozoa. CRISPR-Cas9 was then injected into one-cell zygotes. Embryos at the 4-8-cell stage were collected for genetic analysis. Injection during $\mathrm{S}$-phase resulted in mosaic embryos consisting of non-targeted mutant, targeted NHEJ-repaired and targeted HDR-repaired blastomeres.

Fig. 1d, e and Supplementary Table 1), indicating HDR using the wild-type allele.

The wild-type allele in all iPSC clones analysed remained intact, demonstrating high fidelity of sgRNAs.

We also directly compared CRISPR-Cas9-1 and CRISPR-Cas9-2 in patient iPSCs transfected with preassembled Cas9 ribonucleoproteins (RNPs). Targeted deep sequencing demonstrated that CRISPR-Cas9-1 had higher HDR efficiency (Extended Data Fig. 1f). On-target mutations were not detected in wild-type embryonic stem (ES) cells (H9) carrying both wild-type MYBPC3 alleles, demonstrating high specificity of CRISPR-Cas9-1. On the basis of these outcomes, we selected CRISPR-Cas9-1 (hereafter referred to as CRISPR-Cas9), with higher efficiency of HDR-based gene correction, for subsequent studies.

\section{HDR efficiency in heterozygous MYBPC $3^{\triangle G A G T}$ zygotes}

We next evaluated targeting outcomes in human zygotes produced by fertilizing healthy donor oocytes with sperm from the patient heterozygous for the MYBPC3 mutation. As direct introduction of Cas9 protein is more efficient than using a plasmid ${ }^{9,19}$, recombinant Cas9 protein microinjection was adopted, injecting a mixture of sgRNA, Cas9 protein and ssODN DNA into the cytoplasm of pronuclear stage zygotes $18 \mathrm{~h}$ after fertilization. Injected zygotes and intact controls were cultured for 3 days before each embryonic blastomere was isolated and individually analysed by sequencing (Fig. 1). Cytoplasmic microinjection of CRISPR-Cas9 was confirmed visually (Supplementary Video 1) and shown to be efficient with a $97.1 \%(68 / 70)$ survival rate after injection and development rates comparable to controls (Extended Data Table 2).

Sequencing of 83 individual blastomeres collected from 19 control embryos revealed that $9(47.4 \%)$ were homozygous wild type $\left(M Y B P C 3^{W T / W T}\right)$ and $10(52.6 \%)$ were heterozygous, carrying the wild-type maternal and mutant paternal alleles (MYBPC $3^{W T / \triangle G A G T}$ ) (Fig. 2a and Supplementary Table 2). This distribution was expected and confirms that the heterozygous patient sperm sample contained equal numbers of wild-type and mutant spermatozoa with similar motility and fertilization efficiency. 
a

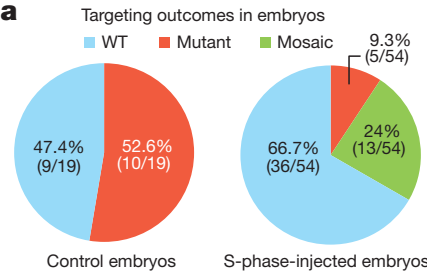

b Targeting outcomes in mosaic embryos
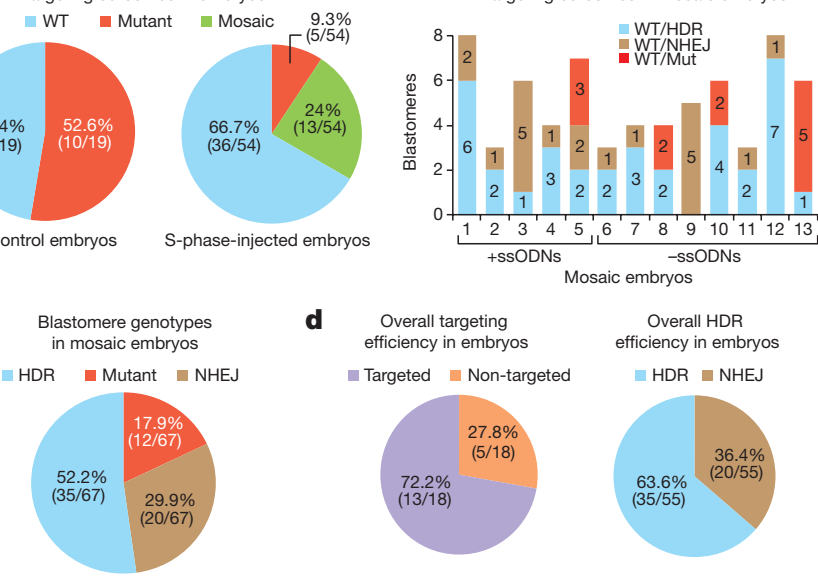

$\mathbf{e}$ Targeting efficiency in
embryos and iPSCs

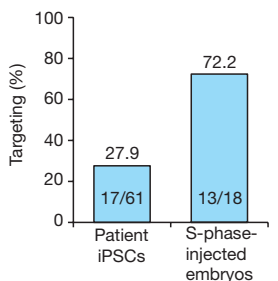

d Overall targeting

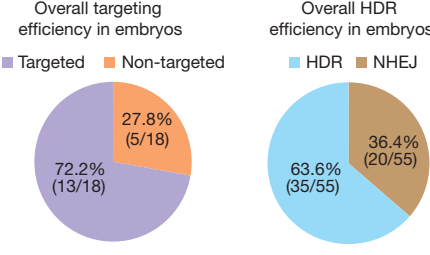

$\square$ Targeted $\quad$ Non-targeted efficiency in embry HDR $\approx$ NHEJ (n)

f Yield of WT/WT embryos

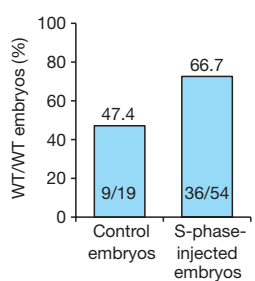

Figure 2 | Gene targeting and HDR efficiency in S-phase-injected human embryos. a, Gene targeting efficiency in zygote, S-phase-injected embryos. b, Blastomere genotyping outcomes in mosaic embryos. c, Various blastomere genotypes in mosaic embryos. d, Overall targeting and HDR efficiency in S-phase-injected embryos. e, Targeting efficiency in patient iPSCs and S-phase-injected embryos. f, Yield of WT/WT embryos in control $(n=19)$ and S-phase-injected $(n=54)$ embryos. For source data, see Supplementary Tables 2 and 3.

Among 54 CRISPR-Cas9-injected embryos, 36 (66.7\%) were uniformly homozygous for the wild-type allele with each blastomere containing MYBPC $3^{W T / W T}$, while $18(33.3 \%)$ were uniform or mosaic heterozygous (Fig. 2a and Supplementary Table 3). In this group of 18, 5 embryos were uniformly heterozygous with each blastomere containing the intact wild-type and intact mutant allele (MYBPC $3^{W T / \triangle G A G T}$ ) and 13 were mosaic, each containing blastomeres carrying more than one genotype (Fig. 2a and Supplementary Table 3). Each mosaic embryo contained at least one heterozygous blastomere with wildtype and either the intact $\triangle$ GAGT deletion or the $\triangle$ GAGT deletion plus additional indels, suggesting that these embryos originated from heterozygous (MYBPC $3^{W T / \triangle G A G T}$ ) zygotes after fertilization by mutant sperm (Fig. 2b and Extended Data Table 3). Remarkably, the majority of the remaining sister blastomeres in eight mosaic embryos (numbers 1, 2, 4, 6, 7, 10, 11 and 12 in Fig. 2b) were homozygous for the wild-type allele (MYBPC3 $\left.3^{W T / W T}\right)$. Overall, 52.2\% (35/67) of individual blastomeres within mosaic embryos were homozygous MYBPC $3^{W T / W T}$ (Fig. 2c and Supplementary Table 3). As these embryos originated from $M Y B P C 3^{W T / \triangle G A G T}$ zygotes, individual blastomeres are likely to have repaired the $M Y B P C 3^{\triangle \mathrm{GAGT}}$ deletion by HDR using the maternal wild-type allele as a template instead of the injected ssODNs. This conclusion was corroborated by the observation that correction occurred in blastomeres of mosaic embryos not injected with ssODNs (Fig. 2b). Among the other genotypes, four mosaic embryos (numbers 5, 8, 10 and 13 in Fig. 2b) contained blastomeres with an intact, mutant allele (MYBPC $3^{W T / \triangle G A G T}$ ), albeit with most $(29.9 \%)$ also containing additional small deletions ( $1-20$ bp length, $n=16)$ or insertions ( $1 \mathrm{bp}, n=3)$ adjacent to the DSB site (MYBPC $\left.3^{W T / \Delta G A G T-i n d e l}\right)$, characteristic of NHEJ. One blastomere carried a 10-bp deletion and a 5-bp insertion (Extended Data Table 3 and Supplementary
Table 3) and mosaic embryo \#9 displayed four various NHEJ genotypes in its blastomeres, suggesting that targeting and NHEJ repair had occurred independently multiple times after the first zygotic division.

The overall targeting efficiency in human embryos was $72.2 \%$ $(13 / 18)$, much higher than in iPSCs exposed to the same construct at $27.9 \%(17 / 61)$ (Fig. 2d, e), probably owing to more efficient delivery of the CRISPR-Cas 9 constructs by zygote microinjection as compared to transfection in iPSCs. Even more remarkably, the majority of targeted blastomeres $(63.6 \%, 35 / 55)$ resolved the DSBs by HDR using the wild-type allele, also markedly different from what was seen in iPSCs (Fig. 2d and Extended Data Fig. 2a). We did not find any evidence of HDR using exogenous ssODN, suggesting that HDR is guided exclusively by the wild-type maternal allele.

As HDR calculations are based on mosaic embryos only, it is probable that some targeted heterozygous $\left(M Y B P C 3^{W T / \triangle G A G T}\right)$ zygotes repaired the mutant allele in all blastomeres using the wild-type template $\left(M Y B P C 3^{W T / W T}\right)$. These HDR-repaired, uniform embryos would be indistinguishable from their wild-type homozygous counterparts, thereby increasing the portion of $M Y B P C 3^{W T / W T}$ embryos in the CRISPR-Cas9 injected group. Indeed, 66.7\% (36/54) of injected embryos were homozygous WT/WT, a substantial increase over the WT/WT yield $(47.4 \%, 9 / 19)$ in control non-injected embryos (Fig. 2f). Similar to observations in iPSCs, the wild-type allele was intact in all human embryos, corroborating the specificity of the sgRNA.

In summary, these results clearly demonstrate that CRISPR-Cas9 gene targeting in human zygotes is exceptionally efficient and that DSBs in the mutant paternal allele were predominantly repaired through HDR. Furthermore, HDR was exclusively directed by the homologous wild-type allele present on the maternal chromosome. This outcome suggests that human embryos employ different DNA repair mechanisms than do somatic or pluripotent cells, probably reflecting evolutionary requirements for stringent control over genome fidelity in the germline.

\section{Targeting gametes eliminates mosaicism}

Mosaicism in gene-targeted human embryos is unacceptable in clinical applications. The presence even of a single mutant blastomere within a mosaic embryo would make detection by PGD problematic; therefore, molecular mechanisms responsible for mosaicism were investigated. Analysis of targeting outcomes in the majority of mosaic, zygote-injected human embryos revealed only two different genotypes $\left(M Y B P C 3^{W T / H D R}\right.$ and MYBPC $3^{W T / \triangle G A G T-i n d e l}$, or MYBPC $3^{W T / H D R}$ and $M Y B P C 3^{W T / \triangle G A G T}$; Fig. $2 \mathrm{~b}$ and Extended Data Table 3). Embryos \#5 and \#9 were the exceptions, containing three or more genotypes (Extended Data Table 3). These findings suggest that CRISPR-Cas9 targeted at least two mutant sperm alleles despite injection into the zygote. There are two different possible explanations for this outcome: 1) at the time of injection, a zygote had completed the S-phase of the cell cycle with DNA replication and already produced two mutant alleles ${ }^{20} ; 2$ ) CRISPR-Cas9 remained active, continuing to target after zygotic division.

We hypothesized that both situations could be abrogated if CRISPRCas9 was co-injected with sperm into the M-phase oocyte during fertilization by intracytoplasmic sperm injection (ICSI), allowing genome editing to occur when the sperm undoubtedly still contains a single mutant copy ${ }^{21}$. In addition, the extended time of exposure to MII cytoplasm could allow CRISPR-Cas9 components to degrade before DNA replication resulted in two or more mutant alleles (Fig. 3a). Therefore, CRISPR-Cas9 was mixed with the sperm suspension and co-injected into 75 MII oocytes during ICSI (Supplementary Video 1); no difference was observed in the survival, fertilization and cleavage rates between CRISPR-Cas9-injected and intact control oocytes (Extended Data Table 2). At day 3 after fertilization, embryos were disaggregated and each individual blastomere was analysed as 
a
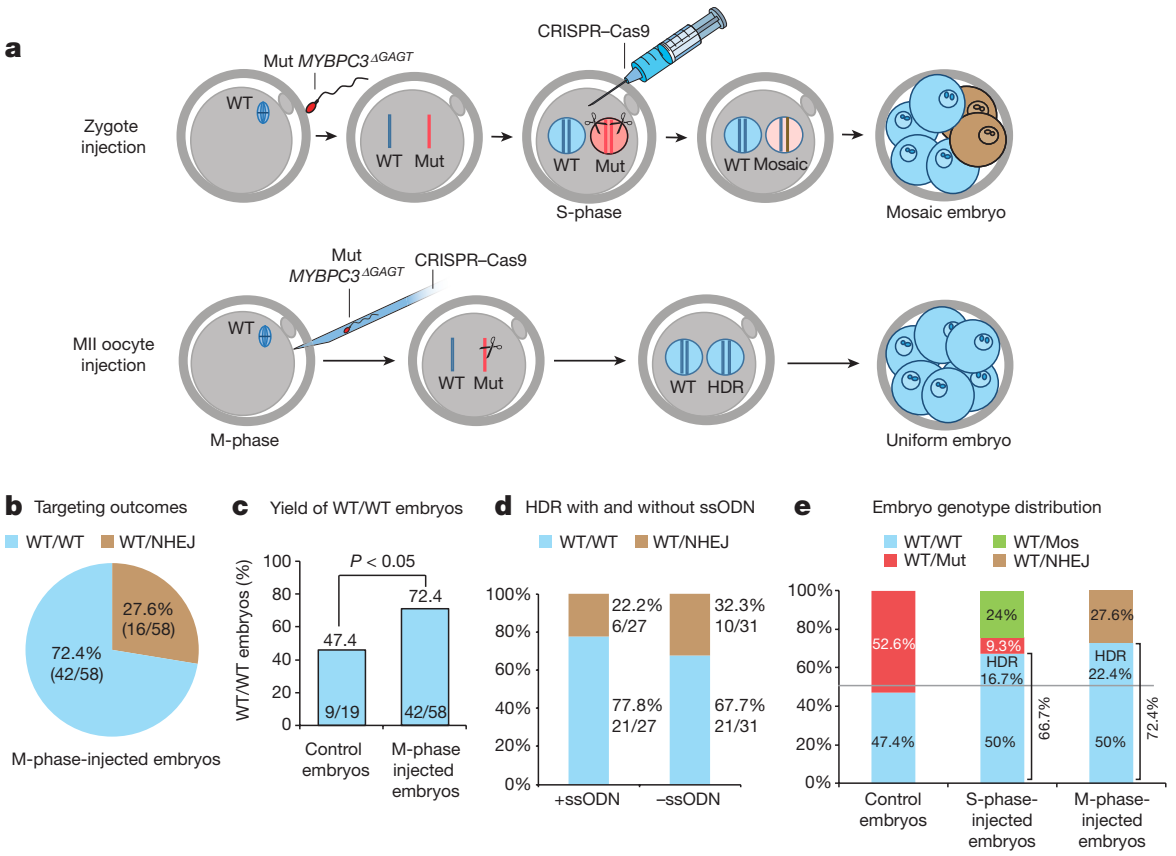

Figure 3 | Gene correction in M-phase-injected human embryos. a, Schematic of $M Y B P C 3^{\triangle \mathrm{GAGT}}$ gene targeting in MII oocytes. CRISPRCas9 was co-injected with sperm into MII oocytes during ICSI. This allows genome editing to occur when a sperm contains a single mutant copy and eliminates mosaicism. $\mathbf{b}$, Targeting efficiency in M-phase-injected embryos. c, Yield of WT/WT embryos in control $(n=19)$ and

described above for S-phase-injected zygotes. Blastomeres from 16 of 58 (27.6\%) M-phase-injected embryos were uniformly heterozygous, carrying an intact wild-type maternal allele along with NHEJ-repaired mutant paternal sequences carrying various indels (MYBPC $3^{\text {WT/ } \triangle G A G T-i n d e l}$ ) (Fig. $3 \mathrm{~b}$ and Supplementary Table 4 ). The remaining 42 (72.4\%) were $M Y B P C 3^{W T / W T}$. Of these, the vast majority $(41 / 42)$ were uniformly homozygous embryos consisting of blastomeres carrying indistinguishable $M Y B P C 3^{W T / W T}$ alleles. Interestingly, the remaining embryo (M2-WT42) contained four blastomeres with MYBPC $3^{W T / W T}$ but HDR-repaired with ssODN, while the other three sister blastomeres were $M Y B P C 3^{W T / W T}$, suggesting HDR using the maternal wild-type allele (Supplementary Table 4). No heterozygous blastomeres with intact mutant alleles (MYBPC $\left.3^{W T / \triangle G A G T}\right)$ were detected, indicating $100 \%$ targeting efficiency in the $\mathrm{M}$-phase-injected group compared to $72.2 \%$ efficiency in the S-phase-injected zygotes (Fig. $2 \mathrm{~d}$ and Fig. 3b). More importantly, all sister blastomeres in all but one embryo carried identical genotypes, indicating a marked reduction in mosaicism in M-phase-injected embryos. The only mosaic embryo had all blastomeres repaired by HDR (either wild-type or ssODN as a template). Thus, this embryo, with every blastomere carrying repaired $M Y B P C 3^{W T / W T}$, would be eligible for transfer.

The yield of MYBPC $3^{W T / W T}$ embryos $(72.4 \%, 42 / 58)$ in the $\mathrm{M}$-phase-injected group was significantly higher than in untreated controls (47.4\%, 9/19) (Fig. 3c, $P<0.05$ ), reflecting enhanced targeted correction of the mutant paternal alleles with DSB repair using the wild-type homologous chromosome as a template even in the presence of ssODNs (Fig. 3d). To rule out the possibility that the observed increase in WT/WT embryos in CRISPR-Cas9-injected zygotes and oocytes was due to allele drop-out during PCR and Sanger sequencing, we validated genotypes by independent on-target deep sequencing (Extended Data Fig. 2b). The estimated HDR-based repair and increase in WT/WT embryos for the S-phase-injected and M-phase-injected groups were $16.7 \%$ (9/54) and 22.4\% (13/58), respectively (Fig. 3e). In summary, delivery of CRISPR-Cas9 into MII oocytes provides more efficient targeting than injection into zygotes while eliminating mosaicism.
M-phase-injected $(n=58)$ embryos. Significance established with onetailed Fisher's test. d, HDR outcomes with or without ssODN. e, HDR efficiencies in S- and M-phase-injected embryos compared to controls. In the S-phase-injected group, each mosaic embryo (green) contained blastomeres with different genotypes. For source data, see Supplementary Table 4.

\section{Development and cytogenetics of repaired embryos}

To examine the effect of gene correction on preimplantation development, we cultured CRISPR-Cas9-injected embryos to blastocysts. Similar to intact controls, $72.7 \%$ (16/22) of M-phase-injected embryos developed to the eight-cell stage and $50.0 \%(11 / 22)$ progressed to blastocysts (Student's $t$-test, $P>0.05$ ) (Fig. $4 \mathrm{a}, \mathrm{b}$ ). In an effort to provide additional insights into the developmental competence of gene-corrected blastocysts, and to obtain sufficient cellular material for detailed cytogenetic studies, we established six ES cell lines from CRISPR-Cas9-injected blastocysts and one from controls. On-target analysis revealed that four CRISPR-Cas9-treated ES cell lines (ES-WT1, ES-WT2, ES-WT3 and ES-WT4) and one control cell line (ES-C1) were MYBPC $3^{W T / W T}$ whereas the remaining two CRISPR-Cas9-injected cell lines (ES-Mut1 and ES-Mut2) were MYBPC $3^{W T / \triangle G A G T-i n d e l}$ (Extended Data Fig. 3a). These results corroborate the exceptionally high targeting efficiency of CRISPRCas9 in M-phase-injected human embryos.

Cytogenetic G-banding analysis revealed that ES-WT1, ES-WT4, ES-Mut1 and ES-Mut2 carried normal diploid karyotypes with no evidence of detectable numerical or structural chromosomal rearrangements (Extended Data Fig. 3a). Notably, ES-WT2, ES-WT3 and the control line ES-C1 exhibited a pericentric inversion on chromosome 10 (Extended Data Fig. 3a-d). As both treated and control ES cells showed this chromosomal rearrangement, we reasoned that it was contributed by the sperm and could be inherited. Indeed, analysis of the patient's skin fibroblast-derived iPSCs showed the same inversion, indicating that this inversion was balanced. In summary, CRISPR-Cas9-treated human embryos displayed normal development to blastocysts and ES cells without cytogenetic abnormalities.

\section{Off-target consequences in repaired human embryos}

Apart from the overall targeting and HDR efficacy and mosaicism, one safety concern regarding clinical application of gene correction in human embryos is that CRISPR-Cas9 can induce undesirable off-target mutations at genome regions that are highly homologous to the targeted sequence ${ }^{22-24}$. Therefore, we conducted a comprehensive, whole-genome 

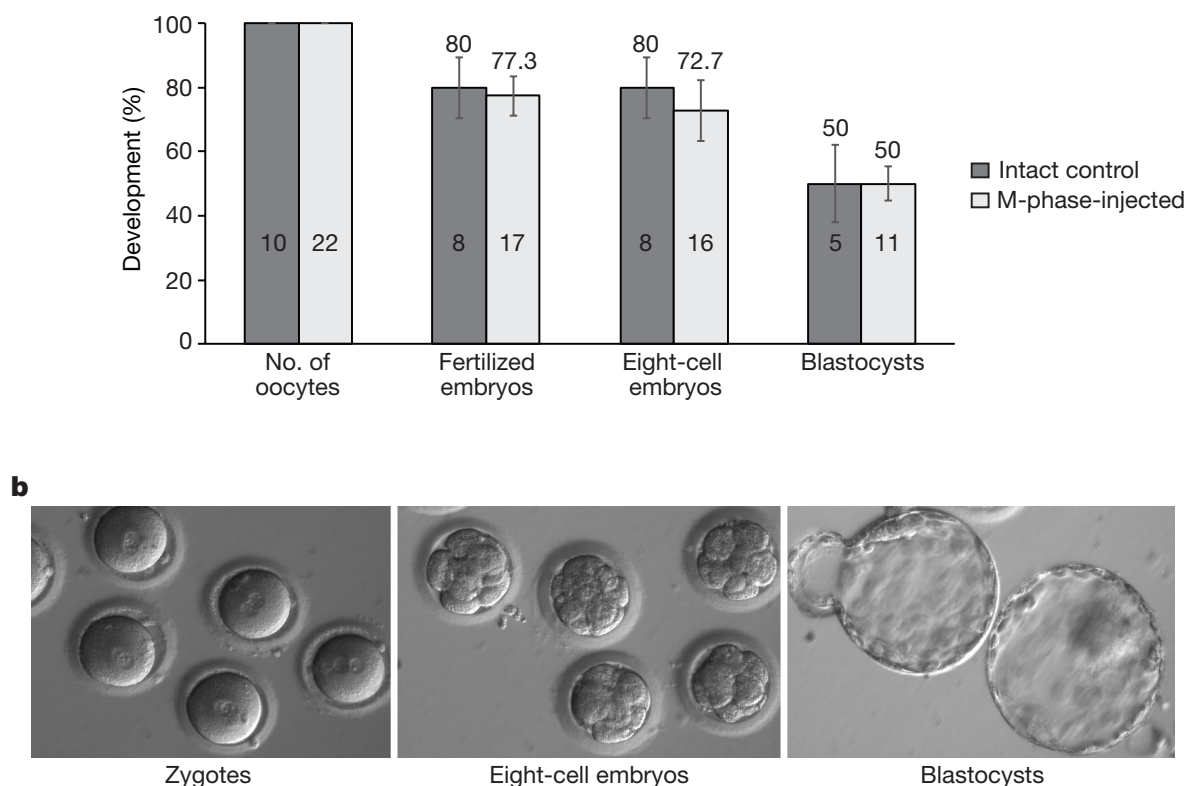

Figure 4 | Preimplantation development of CRISPR-Cas9-injected embryos. a, Fertilization of CRISPR-Cas9-treated $(n=22)$ and control $(n=10)$ MII oocytes and their subsequent development to the eight-cell and blastocyst stage embryos. Number of oocytes/embryos/blastocysts

sequencing (WGS) analysis of the patient's genomic DNA by digested genome sequencing (Digenome-seq) ${ }^{25,26}$. Potential offtarget sequences were identified by digestion of iPSC-derived, cell-free genomic DNA with CRISPR-Cas9 followed by WGS. Sequencing reads of CRISPR-Cas9-digested genomic DNA are vertically aligned at on-/off-target sites in IGV viewer ${ }^{25,27}$. By contrast, undigested genomic sites are aligned in a staggered manner at those loci. In addition, improved Digenome-seq provides DNA cleavage scores for potential off-target sites based on alignment patterns of sequence reads ${ }^{26}$. Digested iPSC DNA produced uniform cleavage patterns at both on-target and potential off-target sites (Extended Data Fig. 4a, b). We identified 16 potential off-target sites with a DNA cleavage score higher than 2.5 (ref. 26) (Extended Data Fig. 5a and Extended Data Table 4). Sequencing analysis of these 16 sites with Web Logo (http://weblogo. berkeley.edu/) confirmed that they are indeed highly homologous to the on-target MYBPC3 mutant allele ${ }^{28}$ (Extended Data Fig. 5b). Furthermore, we identified seven additional sites with DNA cleavage scores of 0.1 or greater and with ten or fewer nucleotides mismatched in the human genome (Extended Data Table 4). Next, we sequenced and analysed all these sites in each individual blastomere from two untreated control embryos (C2 and C10 from Supplementary Table 2), two mosaic S-phase-injected embryos (Mos1 and Mos7), one uniform, non-mosaic S-phase-injected embryo (WT15 from Supplementary Table 3) and two M-phase-injected embryos (M2-WT10 and M2-Mut7 from Supplementary Table 4) (Extended Data Table 5). All on-target indels in each blastomere were corroborated and results were identical to the Sanger sequencing results. In addition, indels were not detected in any blastomeres known to be carrying either intact WT/WT or WT/ Mut alleles at the target site (Extended Data Fig. $5 \mathrm{c}$ and Supplementary Table 5). More importantly, indels were also not detected in 23 off-target loci examined in 28 screened blastomeres (Extended Data Fig. 5d). In selected blastomeres (Extended Data Fig. 5c), we extended off-target screening by WGS. Potential off-target sites were examined by comparing genomic variants found in intact, control embryos (C2.3, C10.2) with those in CRISPR-Cas9-injected embryos (Mos1.1, W15.4, Mos7.2, M2-WT10.1, and M2-Mut7.1) (Extended Data Table 6). After filtering out annotated variants in the dbSNP database, we found 19-71 potential off-target sites with indels in each blastomere obtained from shown in bars; percentage shown above bars. Error bars are mean \pm s.e.m. Significance established using Student's $t$-test. b, Representative images showing normal morphology of CRISPR-Cas9-injected pronuclear stage zygotes, eight-cell embryos and blastocysts.

CRISPR-Cas9-injected embryos (lanes 4 and 7 in Extended Data Table 6). All of these sites contained repeated sequences such as poly-A or poly-GT repeats (lanes 5 and 8 ), suggesting that indels found at these sites were caused by sequencing errors rather than Cas9-catalysed, off-target DNA cleavage. These WGS results support our Digenomeseq conclusions that gene correction did not induce any detectable off-target mutations in selected blastomeres (Extended Data Table 6).

We also investigated whether CRISPR-Cas9 targeting induced global off-target genetic variations and genome instability by performing whole-exome sequencing (WES) in CRISPR-Cas9-treated ES cells and compared the results to those of control ES cells and corresponding egg and sperm donor blood DNA. WES analysis revealed a large number of variants in all samples when compared to the hg19 reference genome (Supplementary Table 6). The majority of these variants were also present in egg or sperm donors and found in the dbSNP and 1000genomes databases. Some variants detected in ES cells showed decreased fractions matching the population hotspots, indicating the potential effect of experimental procedures, including embryo culture and ES cell derivation and culture (Extended Data Fig. 6a and Supplementary Table 6). Three treated ES cell lines and a control line (ES-Mut1, ES-WT1, ES-WT2 and ES-C1) showed similar statistics in all variant categories and were comparable to gamete donor profiles (egg donors 1 and 2, sperm donor). ES-WT3 exhibited an increase in variant numbers but this sample did not have a control sibling ES cell line for comparison (Extended Data Fig. 6b, c and Supplementary Table 6). Next, we investigated potential off-target effects in ES cells and identified a total of 685 potential off-target sites using full sensitive aligner Batmis (V3.00) ${ }^{29}$ (Supplementary Table 7). Variants that were also present in the gamete donors were filtered out as inherited (Supplementary Table 8). Notably, analysis of these sites did not reveal any variants. Taken together, these Digenome-seq, WGS and WES results demonstrate high on-targeting specificity of CRISPR-Cas9 in human embryos without any off-target effects.

\section{Discussion}

DSBs induced by genome editing are primarily resolved via error-prone NHEJ, and such repair approaches are predominantly used to generate gene knockouts in cells and organisms ${ }^{17,30,31}$. By contrast, HDR, 
although occurring at a substantially lower efficiency, is necessary for gene correction, particularly when human germline gene therapy is envisioned. We show here that DSBs in human gametes and zygotes are preferentially resolved using an endogenous HDR mechanism, exclusively directed by the wild-type allele as a repair template. By contrast, HDR efficiency in iPSCs was significantly lower and primarily achieved through an exogenous DNA template. This striking difference implies that human gametes and embryos employ a different DNA damage response system, perhaps reflecting the evolutionary importance of maintaining germline genome integrity ${ }^{32}$. If, as seems likely, gametes and zygotes endure an increased number of DSBs during meiotic recombination and segregation, an efficient genome repair capacity would be critical ${ }^{33}$ and unique zygotic DNA repair machinery might rely entirely on maternal oocyte factors deposited and stored during maturation since zygotes are transcriptionally silent. Recent studies suggest that oocytes might employ an ataxia-telangiectasia mutated (ATM)-mediated DNA damage signalling (DDS) pathway that regulates repair of DSBs via a homologous recombination mechanism ${ }^{34}$. Thus, Cas9-induced DNA breaks are likely to attract the existing native oocyte machinery reserved for repair of meiotic recombinationinduced DSBs. On the basis of our results, it may not even be necessary to provide exogenous oligo templates for gene correction in heterozygous human embryos.

The efficiency of CRISPR-Cas9 was evaluated recently in a mouse study involving a heterozygous dominant mutation in the Crygc gene responsible for an inherited form of cataracts. Some HDR-repaired events used sequences from the wild-type allele from the homologous chromosome ${ }^{12}$. However, HDR via the exogenous oligo template was more frequent, with three of four pups carrying corrected Crygc genes with a DNA sequence from the exogenous oligo and only one from the wild-type allele. In a study involving human heterozygous embryos, HDR was exclusively directed by the exogenous DNA template with no evidence of wild-type allele-based repair ${ }^{35}$. As these results were derived from bulk DNA from whole embryos rather than individual blastomeres, it is possible that instances of HDR via the wild-type allele were overlooked.

Despite remarkable targeting efficiency and high HDR frequency, some CRISPR-Cas9-treated human embryos demonstrated NHEJinduced indels and thus would not be suitable for transfer. Therefore, genome editing approaches must be further optimized before clinical application of germline correction can be considered. Modifications in genome editing by inhibiting NHEJ mechanisms while enhancing HDR pathways have been reported ${ }^{36,37}$. Other approaches have been focused on manipulating the cell cycle or modifying the donor ssDNA design ${ }^{11,30}$. While some of these developments significantly improved HDR outcomes in the context of cultured cells, their relevance to embryonic gene correction remains unknown. In addition, supplementary exposure of human gametes or embryos to small molecules and/or inhibitors may adversely affect embryonic development.

Nonhuman primate studies have demonstrated that CRISPR-Cas9 injection into monkey zygotes can disrupt wild-type genes, with the resultant full-term offspring carrying the mutations and associated phenotypes $^{38,39}$. Similar to the outcomes seen in mice and other animals, genome-edited human preimplantation embryos and newborn monkeys display mosaic targeting genotypes in their cells and tissues, suggesting that DSBs and subsequent repair do not occur at the single mutant allele stage $\mathrm{s}^{3,40,41}$. As discussed above, mosaicism in gene-corrected human embryos would be difficult to detect and would, therefore, pose serious safety issues for possible clinical applications. Modifications involving shortening the half-life of Cas9 activity reduced, but did not completely eliminate, the manifestations of mosaicism in monkey embryos ${ }^{41}$, while introduction of CRISPR-Cas9 into early-stage zygotes reduced mosaicism in mouse embryos ${ }^{42}$.

Importantly, we demonstrate that the delivery of CRISPR-Cas9 into M-phase oocytes abolished mosaicism in cleaving embryos, suggesting that gene targeting and editing efficiencies are strongly associated with
DNA synthesis and the cell cycle phase ${ }^{42}$. The choice of DSB repair using either NHEJ or HDR may be dependent on the cell cycle phase, with HDR restricted to late $S$ and G2 phases when DNA replication is completed and sister chromatids are available as repair templates ${ }^{11}$. In particular, HDR mechanisms were downregulated at the M and early G1 phases, thus favouring NHEJ-induced genome editing ${ }^{43}$. However, we did not observe reduced HDR efficiency even when CRISPR-Cas9 was delivered into MII oocytes at the time of ICSI. One possible explanation is that the DNA repair response is different in germ cell meiotic $\mathrm{M}$ phase compared to mitotic M phase in cultured cells. Alternatively, the DSBs may have occurred at the M or G1 phase, while the HDR repair followed later at the S or G2 phase of the cell cycle.

Extensive reports on potential off-target DNA damage induced by CRISPR-Cas9 beyond the intended targeting region have been published. In particular, Cas9 overexpression via plasmid transfection and subsequent high enzyme concentrations increased off-site targeting ${ }^{9}$. In our studies with human oocytes and zygotes, we used purified recombinant Cas 9 protein instead of plasmid, which may enhance the specificity while shortening enzymatic exposure time, thereby diminishing off-site targeting. Screening by Digenome-seq, WGS and WES did not detect any off-target mutations in multiple individual blastomeres from CRISPR-Cas9-injected human embryos or ES cells. In addition, WES demonstrated maintenance of genome stability after gene correction. CRISPR-Cas9 targeting seen in our investigation was very accurate, providing assurance regarding safety concerns.

Other issues that need to be addressed include the applicability of this gene correction approach to other heterozygous mutations in humans. It may be challenging to repair homozygous mutations in human embryos when both alleles are mutant and wild-type allelebased HDR mechanisms cannot be employed.

As indicated, PGD may be a viable option for heterozygous couples at risk of producing affected offspring. In cases when only one parent carries a heterozygous mutation, $50 \%$ of embryos should be mutant. In contrast, targeted gene correction can potentially rescue a substantial portion of mutant human embryos, thus increasing the number of embryos available for transfer.

Online Content Methods, along with any additional Extended Data display items and Source Data, are available in the online version of the paper; references unique to these sections appear only in the online paper.

\section{Received 28 March; accepted 27 June 2017.}

\section{Published online 2 August 2017.}

1. Antoniou, A. et al. Average risks of breast and ovarian cancer associated with BRCA1 or BRCA2 mutations detected in case series unselected for family history: a combined analysis of 22 studies. Am. J. Hum. Genet. 72, 1117-1130 (2003)

2. Carrier, L., Mearini, G., Stathopoulou, K. \& Cuello, F. Cardiac myosin-binding protein C (MYBPC3) in cardiac pathophysiology. Gene 573, 188-197 (2015).

3. Dhandapany, P. S. et al. A common MYBPC3 (cardiac myosin binding protein C) variant associated with cardiomyopathies in South Asia. Nat. Genet. 41, 187-191 (2009)

4. Struewing, J. P. et al. The risk of cancer associated with specific mutations of BRCA1 and BRCA2 among Ashkenazi Jews. N. Engl. J. Med. 336, 1401-1408 (1997).

5. Maron, B. J. et al. Prevalence of hypertrophic cardiomyopathy in a general population of young adults. Echocardiographic analysis of 4111 subjects in the CARDIA Study. Circulation 92, 785-789 (1995).

6. Schlossarek, S., Mearini, G. \& Carrier, L. Cardiac myosin-binding protein C in hypertrophic cardiomyopathy: mechanisms and therapeutic opportunities. J. Mol. Cell. Cardiol. 50, 613-620 (2011).

7. Hsu, P. D., Lander, E. S. \& Zhang, F. Development and applications of CRISPRCas9 for genome engineering. Cell 157, 1262-1278 (2014).

8. Mali, P. et al. RNA-guided human genome engineering via Cas9. Science 339, 823-826 (2013).

9. Kim, S., Kim, D., Cho, S. W., Kim, J. \& Kim, J. S. Highly efficient RNA-guided genome editing in human cells via delivery of purified Cas9 ribonucleoproteins. Genome Res. 24, 1012-1019 (2014).

10. Cong, L. et al. Multiplex genome engineering using CRISPR/Cas systems. Science 339, 819-823 (2013).

11. Lin, S., Staahl, B. T., Alla, R. K. \& Doudna, J. A. Enhanced homology-directed human genome engineering by controlled timing of CRISPR/Cas9 delivery. eLife 3, e04766 (2014). 
12. Wu, Y. et al. Correction of a genetic disease in mouse via use of CRISPR-Cas9. Cell Stem Cell 13, 659-662 (2013).

13. Guo, X. \& Li, X. J. Targeted genome editing in primate embryos. Cell Res. 25, 767-768 (2015).

14. Wang, H. et al. One-step generation of mice carrying mutations in multiple genes by CRISPR/Cas-mediated genome engineering. Cell 153, 910-918 (2013).

15. Kang, E. et al. Age-related accumulation of somatic mitochondrial DNA mutations in adult-derived human iPSCs. Cell Stem Cell 18, 625-636 (2016)

16. Cho, S. W., Kim, S., Kim, J. M. \& Kim, J. S. Targeted genome engineering in human cells with the Cas9 RNA-guided endonuclease. Nat. Biotechnol. 31, 230-232 (2013).

17. Kim, H. \& Kim, J. S. A guide to genome engineering with programmable nucleases. Nat. Rev. Genet. 15, 321-334 (2014).

18. Jinek, M. et al. A programmable dual-RNA-guided DNA endonuclease in adaptive bacterial immunity. Science 337, 816-821 (2012).

19. Aida, T. et al. Cloning-free CRISPR/Cas system facilitates functional cassette knock-in in mice. Genome Biol. 16, 87 (2015).

20. Capmany, G., Taylor, A., Braude, P. R. \& Bolton, V. N. The timing of pronuclear formation, DNA synthesis and cleavage in the human 1-cell embryo. Mol. Hum. Reprod. 2, 299-306 (1996).

21. Suzuki, T., Asami, M. \& Perry, A. C. Asymmetric parental genome engineering by Cas9 during mouse meiotic exit. Sci. Rep. 4, 7621 (2014).

22. Fu, Y. et al. High-frequency off-target mutagenesis induced by CRISPR-Cas nucleases in human cells. Nat. Biotechnol. 31, 822-826 (2013).

23. Hsu, P. D. et al. DNA targeting specificity of RNA-guided Cas9 nucleases. Nat. Biotechnol. 31, 827-832 (2013).

24. Cho, S. W. et al. Analysis of off-target effects of CRISPR/Cas-derived RNA-guided endonucleases and nickases. Genome Res. 24, 132-141 (2014)

25. Kim, D. et al. Digenome-seq: genome-wide profiling of CRISPR-Cas9 off-target effects in human cells. Nat. Methods 12, 237-243 (2015).

26. Kim, D., Kim, S., Kim, S., Park, J. \& Kim, J. S. Genome-wide target specificities of CRISPR-Cas 9 nucleases revealed by multiplex Digenome-seq. Genome Res. 26, 406-415 (2016).

27. Robinson, J. T. et al. Integrative genomics viewer. Nat. Biotechnol. 29, 24-26 (2011)

28. Schneider, T. D. \& Stephens, R. M. Sequence logos: a new way to display consensus sequences. Nucleic Acids Res. 18, 6097-6100 (1990).

29. Tennakoon, C., Purbojati, R. W. \& Sung, W. K. BatMis: a fast algorithm for k-mismatch mapping. Bioinformatics 28, 2122-2128 (2012),

30. Richardson, C. D., Ray, G. J., DeWitt, M. A., Curie, G. L. \& Corn, J. E. Enhancing homology-directed genome editing by catalytically active and inactive CRISPR-Cas9 using asymmetric donor DNA. Nat. Biotechnol. 34, 339-344 (2016).

31. Doudna, J. A. \& Charpentier, E. The new frontier of genome engineering with CRISPR-Cas9. Science 346, 1258096 (2014).

32. Luo, Y. et al. Hypersensitivity of primordial germ cells to compromised replication-associated DNA repair involves ATM-p53-p21 signaling. PLoS Genet. 10, e1004471 (2014)

33. Lange, J. et al. The landscape of mouse meiotic double-strand break formation, processing, and repair. Cell 167, 695-708 (2016).

34. Titus, S. et al. Impairment of BRCA1-related DNA double-strand break repair leads to ovarian aging in mice and humans. Sci. Transl. Med. 5, 172ra21 (2013).

35. Tang, L. et al. CRISPR/Cas9-mediated gene editing in human zygotes using Cas9 protein. Mol. Genet. Genomics 292, 525-533 (2017).

36. Chu, V. T. et al. Increasing the efficiency of homology-directed repair for CRISPR-Cas9-induced precise gene editing in mammalian cells. Nat. Biotechnol. 33, 543-548 (2015).

37. Maruyama, T. et al. Increasing the efficiency of precise genome editing with CRISPR-Cas 9 by inhibition of nonhomologous end joining. Nat. Biotechnol. 33, 538-542 (2015).

38. Niu, Y. et al. Generation of gene-modified cynomolgus monkey via Cas9/ RNA-mediated gene targeting in one-cell embryos. Cell 156, 836-843 (2014).
39. Kang, Y. et al. CRISPR/Cas9-mediated Dax1 knockout in the monkey recapitulates human AHC-HH. Hum. Mol. Genet. 24, 7255-7264 (2015).

40. Liang, P. et al. CRISPR/Cas9-mediated gene editing in human tripronuclear zygotes. Protein Cell 6, 363-372 (2015).

41. Tu, Z. et al. Promoting Cas9 degradation reduces mosaic mutations in non-human primate embryos. Sci. Rep. 7, 42081 (2017).

42. Hashimoto, M., Yamashita, Y. \& Takemoto, T. Electroporation of Cas9 protein/ sgRNA into early pronuclear zygotes generates non-mosaic mutants in the mouse. Dev. Biol. 418, 1-9 (2016).

43. Orthwein, A. et al. Mitosis inhibits DNA double-strand break repair to guard against telomere fusions. Science 344, 189-193 (2014).

Supplementary Information is available in the online version of the paper.

Acknowledgements We acknowledge the OHSU Institutional Review Board (IRB), Innovative Research Advisory Panel (IRAP), Scientific Review Committee (SRC) and Data Safety Monitoring Committee (DSMC) for oversight and guidance on this study. We thank all study participants for tissue donations; the Women's Health Research Unit staff, IVF laboratory staff, University Fertility Consultants and the Reproductive Endocrinology and Infertility Division in the Department of Obstetrics and Gynecology, OHSU for support and procurement of human gametes; S. Olson and Research Cytogenetics Laboratory at OHSU for cytogenetic analysis of ES cells; S. Cooper from the Wallace Division of Smiths Medical for donating ICSI and gamete manipulation micropipettes; Y. Wang, T. Wu and Y. Shen from BGI-Shenzhen for help with sample preparation, sequencing and data analyses; $\mathrm{M}$. Ku from the $\mathrm{H}$. A. and Mary K. Chapman Charitable Foundations Genomic Sequencing Core of the Salk Institute for next generation sequencing; and E. Aizawa and R. HernandezBenitez from the laboratory of JCIB for assistance. Studies conducted at OHSU were supported by OHSU institutional funds. Work in the laboratory of J.-S.K. was supported by the Institute for Basic Science (IBS-R021-D1). Work in the laboratory of J.C.I.B. was supported by the G. Harold and Leila Y. Mathers Charitable Foundation, the Moxie Foundation and the Leona M. and Harry B. Helmsley Charitable Trust. Work at BGI was supported by the Shenzhen Municipal Government of China (DRC-SZ [2016] 884).

Author Contributions S.M., S.K., Y.Le., N.M.-G. and H.M. conceived the study and designed the experiments. S.-W.P., J.-S.K. and K.S. designed CRISPR-Cas9 constructs. A.K., S.B.H. and S.K. coordinated recruitment of gamete donors. Y.Le. and R.A. derived patient iPSCs and S.-W.P., J.W.,.K.S and J.C.I.B. tested the specificity of the CRISPR-Cas9 systems in patient iPSCs. P.A., D.B., D.M.L., S.A.K. and D.H.W. conducted ovarian stimulation and oocyte retrieval. N.M.-G., Y.Le., D.J. and T.H. performed CRISPR-Cas9 injections, fertilizations, embryo culture and blastomere isolation experiments. N.M.-G., D.J., H.D., C.V.D., Y.Li and E.K. performed DNA extraction, PCR and Sanger sequencing. S.-W.P., A.-R.P., D.K. S.-T.K. and J.-S.K. performed Digenome-seq, WGS and analyses and independently corroborated embryo on-target editing by deep sequencing. J.G., Y.G., X.X. and J.W. performed whole-exome sequencing and analyses. H.M., N.M.-G., S.-W.P., J.W., A.K., D.P.W., S.B.H., J.C.I.B., P.A., J.-S.K., S.K. and S.M. analysed the data and wrote the manuscript.

Author Information Reprints and permissions information is available at www.nature.com/reprints. The authors declare competing financial interests: details are available in the online version of the paper. Readers are welcome to comment on the online version of the paper. Publisher's note: Springer Nature remains neutral with regard to jurisdictional claims in published maps and institutional affiliations. Correspondence and requests for materials should be addressed to S.M. (mitalipo@ohsu.edu), S.K. (kauls@ohsu.edu), J.-S.K. (jskim01@snu.ac.kr),P.A. (amatop@ohsu.edu) or

J.C.I.B. (belmonte@salk.edu).

Reviewer Information Nature thanks F. Lanner and the other anonymous reviewer(s) for their contribution to the peer review of this work. 


\section{METHODS}

Regulations for research on human gametes and embryos. The regulatory framework surrounding the use of human gametes and embryos for this research was based on the guidelines set by the Oregon Health \& Science University (OHSU) Stem Cell Research Oversight Committee (OSCRO). In 2008, OSCRO established policy and procedural guidelines formally defining the use of human embryos and their derivatives at OHSU, informed by the National Academy of Sciences' Guidelines. These policies and guidelines permitted the procurement of gametes and embryos for research purposes, the creation of human embryos specifically for research, genetic manipulation of human gametes and embryos, creation of human embryonic stem cell lines and molecular analyses. Together, OSCRO and the OHSU Institutional Review Board (IRB) worked concurrently to review and monitor applications for research studies involving human embryos at OHSU.

Human embryo and embryonic stem cell research policies and principles at OHSU were vetted over the course of a decade informed by the NAS guidelines, and subsequently affirmed by new guidelines released in 2015 by the Hinxton Group, the International Society for Stem Cell Research (ISSCR), and 2017 recommendations by the NAS and National Academy of Medicine joint panel on human genome editing.

As part of the review process, OHSU convened additional ad hoc committees to evaluate the scientific merit and ethical justification of the proposed study: the OHSU Innovative Research Advisory Panel (IRAP) and a Scientific Review Committee (SRC). Members of both committees were independent and their names were kept confidential from the research team; OHSU Research Integrity supervised all committee meetings, documentation, and formal recommendations. Ethical review. While international discussions were in their infancy, the OHSU Innovative Research Advisory Panel (IRAP) Committee was tasked with deliberating ethical considerations related to using gene correction technology in human embryos for basic research at OHSU. The committee was composed of eleven members from internal and external sources: a lay member, a clinical ObGyn physician, three bioethicists, an OHSU Institutional Ethics committee member, three former OSCRO members, a clinical geneticist, and a clinician. Upon completion of the review, the IRAP recommended allowing this research "with significant oversight and continued dialogue, the use of gene correction technologies in human embryos for the purpose of answering basic science questions needed to evaluate germline gene correction prior to the use in human models," at OHSU. Study oversight. The established track record of the study team to uphold strict confidentiality and regulatory requirements paved the way for full OHSU IRB study approval in 2016, contingent upon strict continuing oversight which includes: a phased scientific approach requiring evaluation of results on the safety and efficacy of germline gene correction in iPSCs before approving studies on human pre-implantation embryos; external bi-annual monitoring of all regulatory documents regarding human subjects; bi-annual Data Safety Monitoring Committee (DSMC) review; and annual continuing review by the OHSU IRB. The DSMC is required to remain active for the length of the approved IRB protocol and consists of four members: a lay member, an ethicist, a geneticist, and a reproductive endocrinologist. This committee conducts full review of all donations, the subsequent uses of these samples, and participant adverse events. The DSMC provides formal recommendations to the study team and IRB at the completion of each meeting. Informed consent. The robust regulatory framework set forth by OHSU clearly specified that informed consent could be obtained only if prospective donors were made aware of the sensitive nature of the study. The consent form clearly presented the scientific rationale for the study; stating (in both the Clinical Research Consent Summary and the Purpose section of the consent form) that gene editing tools will be used on eggs, sperm, and/or embryos to evaluate the safety and efficacy of gene correction for heritable diseases. Additionally, consent form language clearly stated that genetic testing would be conducted in addition to creation of preimplantation embryos and embryonic stem cell lines for in vitro analyses and stored for future use. The incidental discovery of genetic information that might be important to the donors' healthcare is a possible outcome when engaging in this type of research. Informed consent documents provided the donor with the option to receive this information or not. Written informed consent was obtained before all study-related procedures on current, IRB-approved, study-specific consent forms.

Study participants. Healthy gamete donors were recruited locally, via print and web-based advertising. Homozygous and heterozygous adult patients with known heritable MYBPC3 mutations were sought; however, only three adult heterozygous patients were identified by OHSU Knight Cardiovascular Institute physicians and referred to the research team, one of whom agreed to participate in the study.

Controlled ovarian stimulation. Research oocyte donors were evaluated before study inclusion as previously reported; standard IVF protocols and procedures for ovarian stimulation were described previously ${ }^{44}$. Oocyte donation cycles were managed by OHSU Fertility physicians. Immediately following oocyte retrieval, recovered gametes were transferred to the research laboratory. All study-related procedures took place at the OHSU Center for Embryonic Cell and Gene Therapy. Following oocyte retrieval, cumulus-oocyte complexes (COCs) were treated with hyaluronidase to disaggregate cumulus and granulosa cells. Mature metaphase II (MII) oocytes were placed in Global Medium (LifeGlobal, IVFonline) supplemented with $10 \%$ serum substitute supplement (Global $10 \%$ medium) at $37^{\circ} \mathrm{C}$ in $6 \% \mathrm{CO}_{2}$ and covered with tissue culture oil (Sage IVF, Cooper Surgical).

Compensation. All research donors were compensated for their time, effort, and discomfort associated with the donation process at rates similar to those used for gamete donation for fertility purposes.

Intracytoplasmic sperm injection (ICSI). MII oocytes were placed into a $50-\mu$ I micromanipulation droplet of HTF (modified human tubal fluid) with HEPES $10 \%$ medium. The droplet was covered with tissue culture oil. The dish was then mounted on the stage of an inverted microscope (Olympus IX71) equipped with a stage warmer (http://www.tokaihit.com) and Narishige micromanipulators. Oocytes were fertilized by ICSI using frozen and thawed sperm. Fertilization was determined approximately $18 \mathrm{~h}$ after ICSI by noting the presence of two pronuclei and second polar body extrusion.

CRISPR-Cas9 injection into zygote or oocytes. For S-phase injections, zygotes were collected $18 \mathrm{~h}$ after ICSI and placed in a micromanipulation drop. The CRISPR-Cas 9 mixture, containing Cas 9 protein $(200 \mathrm{ng} / \mu \mathrm{l})$, sgRNA $(100 \mathrm{ng} / \mu \mathrm{l})$ and ssODN $(200 \mathrm{ng} / \mu \mathrm{l})$, was then injected into the cytoplasm. Injected zygotes were cultured in Global $10 \%$ medium at $37^{\circ} \mathrm{C}$ in $6 \% \mathrm{CO}_{2}, 5 \% \mathrm{O}_{2}$ and $89 \% \mathrm{~N}_{2}$ for up to 3 days to the 4-8-cell stage. For M-phase injections, CRISPR-Cas9 was co-injected with sperm during ICSI. A single sperm was first washed in a 4- $\mu \mathrm{l}$ drop of mixture containing Cas9 protein, sgRNA, and ssODN as described above.

Blastomere isolation, whole-genome amplification and Sanger sequencing. Zonae pellucidae from 4-8-cell stage embryos were removed by brief exposure to acidic Tyrode solution $\left(\mathrm{NaCl} 8 \mathrm{mg} / \mathrm{ml}, \mathrm{KCl} 0.2 \mathrm{mg} / \mathrm{ml}, \mathrm{CaCl}_{2} .2 \mathrm{H}_{2} \mathrm{O} 2.4 \mathrm{mg} / \mathrm{ml}\right.$ $\mathrm{MgCl}_{2} .6 \mathrm{H}_{2} \mathrm{O} 0.1 \mathrm{mg} / \mathrm{ml}$, glucose $1 \mathrm{mg} / \mathrm{ml}$, PVP $\left.0.04 \mathrm{mg} / \mathrm{ml}\right)$. Zona-free embryos were briefly $(30 \mathrm{~s})$ exposed to a trypsin solution $(0.15 \%$ in EDTA containing $\mathrm{Ca}$ - and Mg-free PBS) before manual disaggregation into single blastomeres with a small bore pipette. A total of 830 blastomeres were isolated from 131 embryos, including 19 from control, 54 from zygote-injected and 58 from $\mathrm{M}$-phase-injected groups. Individual blastomeres were transferred into $0.2-\mathrm{ml}$ PCR tubes containing $4 \mu \mathrm{l} \mathrm{PBS}$ and placed into a freezer at $-80^{\circ} \mathrm{C}$ until further use. Whole-genome amplification was performed using a REPLI-g Single Cell Kit (Qiagen). Successful amplification was evaluated by PCR for MYBPC3 and Sanger sequencing. Briefly, amplified DNA was diluted $1 / 100$ and the on-target region for MYBPC3 was amplified using a PCR Platinum SuperMix High Fidelity Kit (Life Technologies) with primer set: F 5'-CCCCCACCCAGGTACATCTT-3' and R 5'-CTAGTGCACAGTGCATAGTG-3'. PCR products of 534 base pairs $(\mathrm{bp})$ were purified, Sanger sequenced and analysed by Sequencher v5.0 (GeneCodes). Of 830 blastomeres, $730(88 \%)$ resulted in successful libraries and produced PCR products for MYBPC3 while the remaining 100 blastomeres (12\%) failed to generate PCR products and were excluded from the study.

iPSC derivation and transfection with CRISPR-Cas9. Patient iPSCs were derived from skin fibroblasts with a CytoTune-iPS Reprogramming Kit (Life Technologies), according to the manufacturer's protocol. Cell lines were cultured in mTeSR 1 medium (STEMCELL technology) at $37^{\circ} \mathrm{C}$ in a humidified atmosphere containing $5 \% \mathrm{CO}_{2}$. To test CRISPR-Cas $9,2 \times 10^{5}$ iPSCs were dissociated into single cells (using Accutase from STEMCELL technology, or TrypLe from Invitrogen). For the CRISPR-Cas9-1 construct (in the J.-S.K. laboratory), Cas9 expression plasmid (p3 s-Cas9HC, $2.4 \mu \mathrm{g}$ ), sgRNA expression plasmid (pU6-sgRNA, 1.6 $\mu \mathrm{g}$ ) and ssODN-1 (100 pmol, IDT) were transfected into iPSCs using an Amaxa P3 Primary Cell 4D-Nucleofector Kit (Program CB-150) according to the manufacturer's protocol. Three days after transfection, $\sim 5,000$ cells were plated onto a Matrigel-coated culture dish and cultured for clonal propagation and individual clone selection. For the CRISPR-Cas9-2 construct (in the J.C.I.B. laboratory), Cas9 expression plasmid (pCAG-1BPNLS-Cas9-1BPNLS, 15ug), sgRNA expression plasmids (pCAGmCherry-MYBPC3gRNA, 15ug) and $30 \mu \mathrm{g} s \mathrm{ODN}-2$ (IDT) were co-transfected by electroporation using the BioRad Gene Pulser II (a single 320-V, 200- $\mu \mathrm{F}$ pulse at room temperature) with a $0.4-\mathrm{cm}$ gap cuvette. Cells were plated at high density on 6-well plates coated with Matrigel. Two to three days after electroporation, iPSCs were harvested and subjected to clonal selection. All cell lines were negative for mycoplasma contamination. For direct comparisons of CRISPR-Cas9-1 and CRISPR-Cas9-2, Cas9 RNP complexes, composed of the recombinant Cas 9 protein $(15 \mu \mathrm{g})$ and sgRNA $(20 \mu \mathrm{g})$, were co-transfected with ssODN-1 (50-200 pmol, IDT) into iPSCs $\left(2 \times 10^{5}\right.$ cells) via electroporation as described above. Three days after transfection, indel and HDR efficiencies were analysed by targeted deep sequencing. 
Recombinant Cas9 protein and in vitro transcription of sgRNA. Recombinan Cas9 protein was purchased from ToolGen, Inc. The sgRNA was synthesized by in vitro transcription using T7 polymerase (New England Biolabs) as described previously ${ }^{45}$. In brief, sgRNA templates were generated by annealing and extension of two oligonucleotides (Extended Data Table 1). Then, in vitro transcription was performed by incubating sgRNA templates with T7 RNA polymerase supplemented with NTPs (Jena Bioscience) and RNase inhibitor (New England Biolabs) overnight at $37^{\circ} \mathrm{C}$. In vitro transcribed RNA was then treated with DNase I (New England Biolabs) for $30 \mathrm{~min}$ at $37^{\circ} \mathrm{C}$, and purified using MinElute Cleanup kit (Qiagen).

Targeted deep sequencing, genomic DNA cleavage, WGS and Digenome sequencing. To analyse HDR and NHEJ frequencies, on-target and off-target regions were amplified using Phusion polymerase (New England Biolabs). PCR amplicons were subjected to paired-end sequencing using Illumina Miniseq. Cas-analyzer was used for analysing indel and HDR frequencies ${ }^{46,47}$. The primers used for amplification are listed in Extended Data Table 5. Genomic DNA was isolated from patient iPSCs using a DNeasy Tissue Kit (Qiagen). Digenome-seq was performed as described ${ }^{25,26}$. In brief, $20 \mu \mathrm{g}$ genomic DNA was cleaved by incubating recombinant Cas9 protein $(16.7 \mu \mathrm{g})$ and in vitro transcribed sgRNA $(12.5 \mu \mathrm{g})$ in $1 \times \mathrm{NEB}$ buffer 3.1 $(100 \mathrm{mM} \mathrm{NaCl}, 50 \mathrm{mM}$ Tris- $\mathrm{HCl}, 10 \mathrm{mM} \mathrm{MgCl} 2,100 \mu \mathrm{g} / \mathrm{ml}$ BSA, $\mathrm{pH} 7.9$ ) at $37^{\circ} \mathrm{C}$ for $3 \mathrm{~h}$. Cas9- and sgRNA-treated genomic DNA was treated with $50 \mu \mathrm{g} / \mathrm{ml}$ RNase A (Sigma Aldrich) at $37^{\circ} \mathrm{C}$ for $30 \mathrm{~min}$, and purified with a DNeasy Tissue Kit (Qiagen). WGS and Digenome sequencing were performed as described previously ${ }^{25,26}$. In brief, $1 \mu \mathrm{g}$ genomic DNA was fragmented and ligated with adaptors using TruSeq DNA libraries. DNA libraries were subjected to WGS using an Illumina HiSeq X Ten Sequencer at Macrogen $(30 \times$ to $40 \times)$. The sequence file was aligned to the human reference genome hg19 from UCSC with the following mapping program and parameters using Isaac aligner ${ }^{48}$ : base quality cutoff, 15; keep duplicate reads, yes; variable read length support, yes; realign gaps, no; and adaptor clipping, yes (adaptor: AGATCGGAAGAGC*, *GCTCTTCCGATCT). In vitro DNA cleavage sites were identified computationally using a DNA cleavage scoring system described previously ${ }^{26}$. Indel frequencies of 23 genomic loci with DNA cleavage score above the 0.1 cutoff value were individually examined in individual blastomeres by targeted deep sequencing. Primers for detecting indel frequencies of Digenome-seq captured sites are listed in Extended Data Table 5.

Analysis of off-target effects in CRISPR-Cas9-injected human embryos by WGS. WGS was performed using an Illumina HiSeq X Ten sequencer with a sequencing depth of $30 \times$ to $40 \times$ (Macrogen, South Korea). Sequences from each blastomere were processed to obtain total variants (lane 1 in Extended Data Table 6) using the Isaac variant calling program ${ }^{48}$. Annotated variants, including dbSNPs and all novel SNPs (substitution changes), were filtered out, and novel indel sites were identified (lane 2 in Extended Data Table 6). Cas-OFFinder ${ }^{46}$ was used to extract potential off-target sequences that differed from the on-target sequence by up to 7 -nucleotide mismatches or up to 5 -nucleotide mismatches with a DNA bulge of up to 2 nucleotides. Indel sites found in each blastomere were compared to homologous sites identified by Cas-OFFinder and potential off-target sites were identified (lanes 3 and 6 in Extended Data Table 6). Then, we excluded potential off-target sites, which were found in intact control embryos (C2.3 and C10.2, lanes 4 and 7 in Extended Data Table 6). Finally, we determined whether each of these potential off-target sites was caused by CRISPR-Cas 9 by inspecting sequences with Integrative Genomics Viewer ${ }^{27}$ (IGV, lanes 5 and 8 in Extended Data Table 6).
Whole-exome sequencing and data analyses. Whole-exome sequencing (WES) was performed using genomic DNAs isolated from peripheral blood of the sperm donor and two egg donors (egg donor1 and egg donor2) and ES cells derived from individual human embryos (ES-WT1, ES-Mut1 and ES-C1 were from egg donor1; ES-WT2 and ES-WT3 were from egg donor2). ES-WT1, ES-WT2 and ES-WT3 were from treated wild-type embryos. ES-C1 was from an untreated wild-type embryo. ES-Mut1 was from a treated heterozygous mutant embryo. Sequencing libraries were prepared according to the instructions for Illumina library preparation. Exome capture was done using an Agilent V5 chip. Sequencing was done on an Illumina Hiseq 4000 platform with paired-end 101 (PE101) strategy at a depth of $100 \times$. All sequencing data were first processed by filtering adaptor sequences and removing low quality reads or reads with a high percentage of $\mathrm{N}$ bases using SOAPnuke (1.5.2) software (http://soap.genomics.org.cn/) developed by BGI, and clean reads were generated for each library. Clean data were paired-end aligned using the Burrows-Wheeler Aligner (BWA) program version 0.7.12 to the human genome assembly hg19. Duplicate reads in alignment BAM files were identified using MarkDuplicates in Picard (1.54). The alignment results were processed by RealignerTargetCreator, IndelRealigner and BaseRecalibrator modules in GATK (3.3.0). Variant detection was performed using HaplotypeCaller tool in GATK. SNV and indel information was extracted and filtered by VQSR in GATK and annotated by AnnoDB (v3).

The guide sequence (GGGTGGAGTTTGTGAAGTAT) was aligned to the human genome assembly hg19 to identify potential off-target sites by full sensitive aligner Batmis (V3.00), allowing a maximum of five mismatches globally and a maximum of two mismatches in the core region (12 bp adjacent to the PAM site). Inherited variants from parents and all novel SNPs (substitution changes) were filtered out, and novel indels located within the off-target site plus flanking 20-bp region were defined as off-target variants.

Statistical analyses. Student's $t$-test was used for the comparisons in Fig. 4a. Onetailed Fisher's test was used for the comparisons in Fig. 2f, Fig. 3c, and Extended Data Fig. 1e. One-way ANOVA with Bonferroni test was used for the comparisons in Extended Data Table 2. A P value less than 0.05 was considered significant. No statistical methods were used to predetermine sample size. The experiments were randomized and the investigators were blinded to allocation during experiments and outcome assessment whenever possible.

Data availability. The sequencing data sets, including WGS, WES and Digenomeseq, generated during the current study are not being made publicly available owing to concerns that the data could reveal the research participant's genetic identity, and revealing the identity would be against the participant's wishes and consent. However, the data will be made available to researchers from the corresponding author on reasonable request, dependent upon OHSU IRB approval.

44. Tachibana, M. et al. Towards germline gene therapy of inherited mitochondrial diseases. Nature 493, 627-631 (2013).

45. Kim, J. M., Kim, D., Kim, S. \& Kim, J. S. Genotyping with CRISPR-Cas-derived RNA-guided endonucleases. Nat. Commun. 5, 3157 (2014).

46. Bae, S., Park, J. \& Kim, J. S. Cas-OFFinder: a fast and versatile algorithm that searches for potential off-target sites of Cas9 RNA-guided endonucleases. Bioinformatics 30, 1473-1475 (2014).

47. Park, J., Lim, K., Kim, J. S. \& Bae, S. Cas-analyzer: an online tool for assessing genome editing results using NGS data. Bioinformatics 33, 286-288 (2017).

48. Raczy, C. et al. Isaac: ultra-fast whole-genome secondary analysis on Illumina sequencing platforms. Bioinformatics 29, 2041-2043 (2013). 
a

CRISPR-Cas9-1 (sgRNA(19bp)-Cas9)

MYBPC3

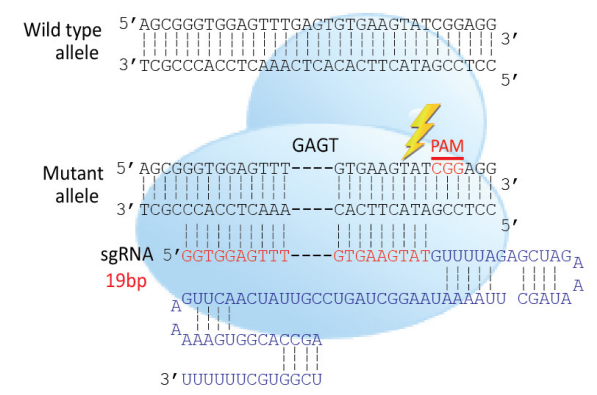

5' ---GGAGTTTGAGTGCGAGGTATCG---3' ssODN-1 (200 mer)

C

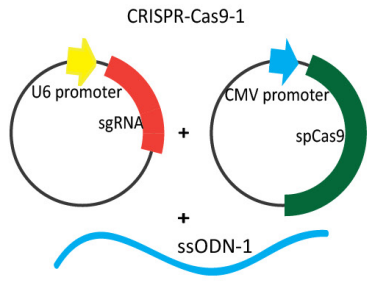

CRISPR-Cas9-2

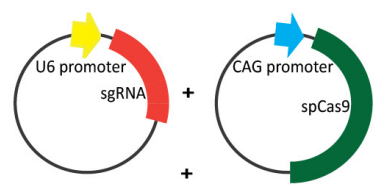

SSODN-2

d

Heterozygous mutant iPSCs

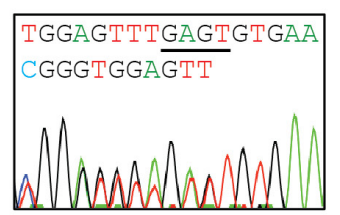

Electroporation

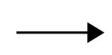

HDR/SSODN-2 iPSCS

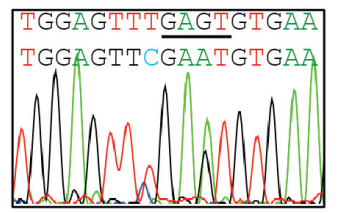

CRISPR-Cas9-2 (sgRNA(20bp)-Cas9)

МYBPC3

Wild type 5'AGGGGGTGGATTTGAGTGTGAAGTATCGGAGG $3^{\prime}$

CTCC 5

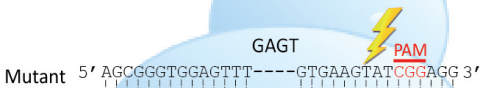

Mutant 5 AGCGGGTGGAGTTT---GTGAAGTATCGGAGG 3

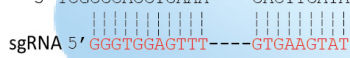

GAGTATGUUUAGAGCUAG

20bp GUUCAACUAUUGCCUGAUCGGAaUAAAAUU CGAUA

A AAAGUGGCACCGA

3' UUUUUUCGUGGCU

BstBI

5' ---GGAG PT CGAATGTGAAGTATCG---3' ssODN-2 (100 mer)

Patient iPSCs

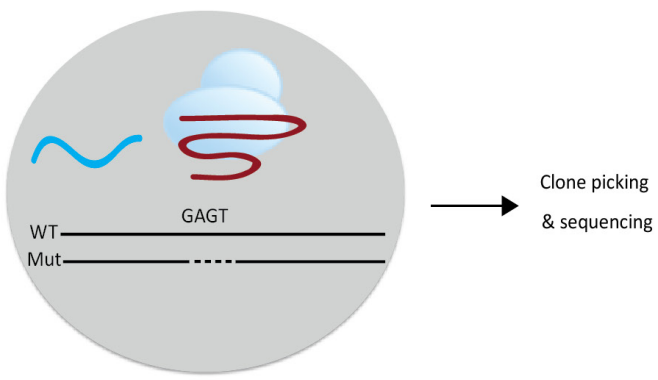

e

\begin{tabular}{ccccccc}
\hline CRISPR-Cas9 & Clones analyzed & Clones targeted & $\begin{array}{c}\text { Clones with NHEJ } \\
(\%)\end{array}$ & $\begin{array}{c}\text { Clones corrected } \\
\text { (HDR/ssODN) }\end{array}$ & $\begin{array}{c}\text { Clones corrected } \\
\text { (HDR/WT) }\end{array}$ & $\begin{array}{c}\text { HDR efficiency } \\
\text { HDR }\end{array}$ \\
\hline 1 & 61 & $17(27.9 \%)^{*}$ & $10(58.8 \%)$ & 7 & 0 & $41.2 \%(7 / 17)$ \\
2 & 175 & $23(13.1 \%)$ & $20(86.9 \%)$ & 2 & 1 & $13 \%(3 / 23)$ \\
\hline
\end{tabular}

$\mathbf{f}$
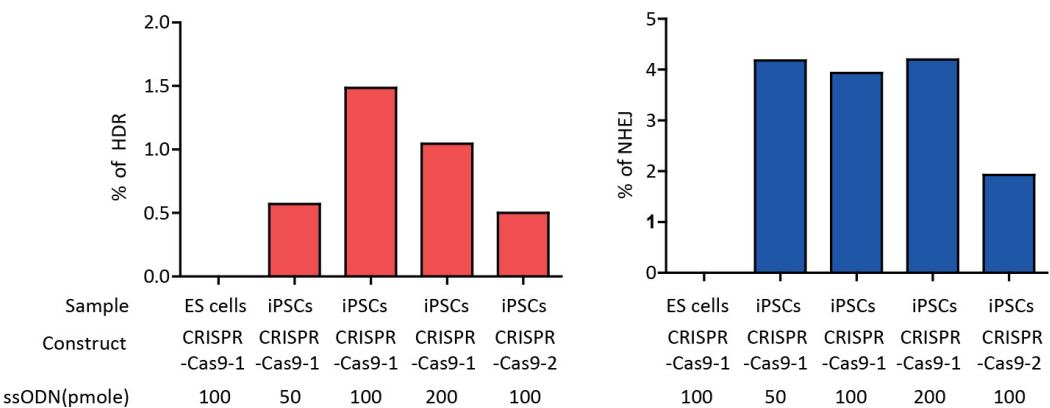

Extended Data Figure 1 | See next page for caption. 
Extended Data Figure 1 | CRISPR-Cas9 design and testing in patient iPSCs. a, b, Schematic of CRISPR-Cas9-1 and CRISPR-Cas9- 2 constructs. Both systems consist of a single-chain chimaeric sgRNA designed to target the $M Y B P C 3^{\triangle G A G T}$ deletion and Cas9 protein. Exogenous single-stranded oligodeoxynucleotide (ssODN) templates encoding homology arms to the targeted region were designed for each system to facilitate HDR. Synonymous single-nucleotide substitutions were introduced into each ssODN template as indicated by red letters. Underline in la represents wild-type sequence of 4-bp deletion in the ssODN-1. In addition, the ssODN-2 nucleotide substitutions provide a restriction enzyme (BstBI) recognition site as indicated with black open box. c, Patient iPSCs were transfected with CRISPR-Cas9 plasmids by electroporation and individual single iPSC clones were analysed. d, Representative chromatographs showing untargeted iPSC clone with heterozygous mutant (left), targeted iPSC clone with gene corrected via HDR using ssODN-2 as repair template (middle) and targeted iPSC clone with gene corrected via HDR using wildtype sequence as template. e, Targeting and HDR efficiency comparison between CRISPR-Cas9-1 and CRISPR-Cas9-2. The asterisk indicates a significantly higher targeting efficiency of CRISPR-Cas9-1 compared to CRISPR-Cas9-2 $(P<0.05)$ by one-tailed Fisher's test. For source data, see Supplementary Table 1.f, HDR and NHEJ efficiency in wild-type ES cells (H9) and patient iPSCs transfected with preassembled Cas9 ribonucleoproteins (RNPs). 
a
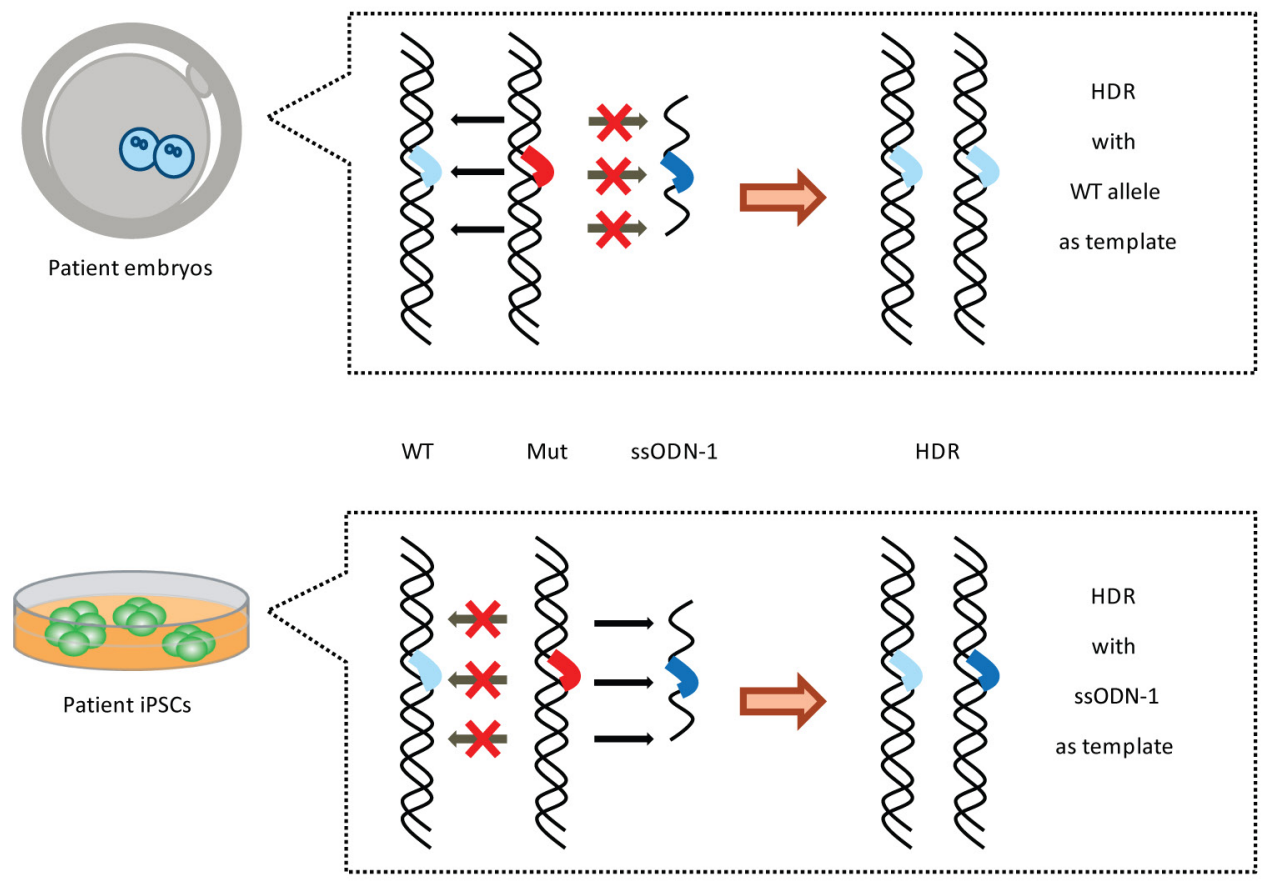

HDR efficacy in

embryos and iPSCs
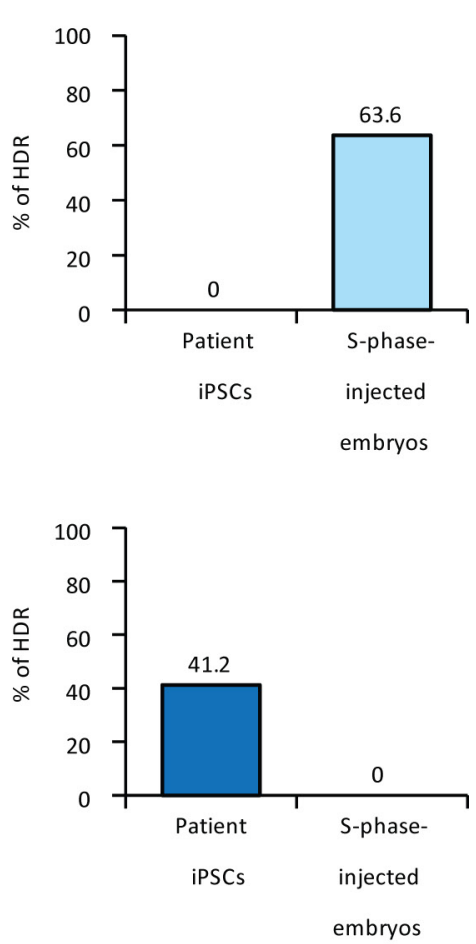

b

Verification of blastomere genotypes by on-target deep sequencing

\begin{tabular}{lccccc}
\hline Treatment & $\begin{array}{c}\text { No. of blastomeres } \\
\text { or samples }\end{array}$ & $\begin{array}{c}\text { Genotypes by } \\
\text { Sanger }\end{array}$ & Total reads & $\begin{array}{c}\text { WT reads } \\
(\%)\end{array}$ & $\begin{array}{c}\text { Mutant reads } \\
(\%)\end{array}$ \\
\hline Intact control WT embryos & 8 & WT/WT & 10007 & $9993(99.9 \%)$ & $14(0.1 \%)$ \\
Intact control mutant embryos & 8 & WT/Mut & 10281 & $4988(48.5 \%)$ & $5293(51.5 \%)$ \\
\hline Zygote injection WT embryos & 14 & WT/WT & 13433 & $13426(99.9 \%)$ & $7(0.1 \%)$ \\
Zygote injection mutant embryos & 16 & WT/Mut & 11583 & $6942(59.9 \%)$ & $4641(40.1 \%)$ \\
Zygote injection mosaic embryos & 8 & WT/HDR & 13193 & $13191(99.99 \%)$ & $2(0.01 \%)$ \\
\hline MIl injection WT embryos & 5 & WT/NHEJ & 9634 & $6330(65.7 \%)$ & $3304(34.3 \%)$ \\
MII injection mutant embryos & 12 & WT/WT & 12613 & $12607(99.9 \%)$ & $6(0.1 \%)$ \\
\hline Oocyte donor blood & 15 & WT/NHEJ & 11016 & $3969(36 \%)$ & $7047(64 \%)$ \\
\hline
\end{tabular}

Extended Data Figure $2 \mid$ Differences in DNA repair responses in human embryos and iPSCs and independent validation of on-target genotyping. a, Different HDR-based DNA repair was observed in patient embryos compared to iPSCs. In human embryos, induced DSBs in the mutant paternal gene were repaired exclusively using the wild-type maternal allele as a template. In striking contrast, HDR in iPSCs was directed by the external ssODN-1 template. b, Verification of blastomere on-target genotypes by deep sequencing. DNA samples from 86 individual blastomeres representing different treatments and controls were sequenced using the MiSeq platform. Total reads, wild-type reads and mutant reads are presented as a mean. Distribution of mutant reads in heterozygous (34.3-64\%) or homozygous (0.01-0.1\%) blastomeres in experimental embryos was similar to controls. 
Origin and genotypes of ES cells derived from CRISPR-Cas9 injected embryos

\begin{tabular}{ccccc}
\hline $\begin{array}{c}\text { ES cell line } \\
\text { designation }\end{array}$ & Treatment & Karyotype & $\begin{array}{l}\text { On target } \\
\text { genotype }\end{array}$ & Egg donor \\
\hline ES-WT1 & M-phase injection & $46, \mathrm{XX}$ & WT/WT & Egg donor 1 \\
ES-WT2 & M-phase injection & $46, \mathrm{XX}$, inv $(10)(\mathrm{p} 11.2 \mathrm{q} 21.2)$ & WT/WT & Egg donor 2 \\
ES-WT3 & M-phase injection & $46, \mathrm{XY}$, inv $(10)(\mathrm{p} 11.2 \mathrm{q} 21.2)$ & WT/WT & Egg donor 2 \\
ES-WT4 & M-phase injection & $46, \mathrm{XX}$ & WT/WT & Egg donor 2 \\
ES-Mut1 & M-phase injection & $46, \mathrm{XX}$ & WT/NHEJ & Egg donor 1 \\
ES-Mut2 & M-phase injection & $46, \mathrm{XX}$ & WT/NHEJ & Egg donor 2 \\
ES-C1 & Intact control & $46, \mathrm{XY}$, inv $(10)(\mathrm{p} 11.2 \mathrm{q} 21.2)$ & WT/WT & Egg donor1 \\
\hline
\end{tabular}

b

ES-WT3

$46, X Y, \operatorname{inv}(10)(p 11.2 q 21.2)$
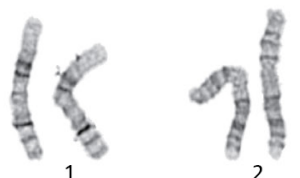

ह 8

6
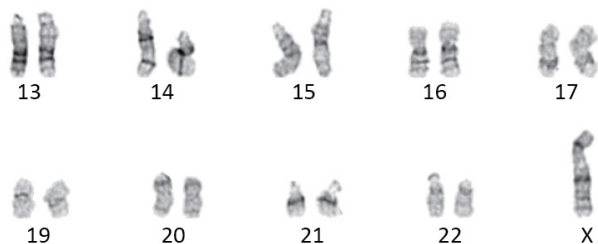

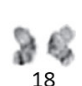

Chromosome 10 $\operatorname{inv}(10)(\mathrm{p} 11.2 \mathrm{q} 21.2)$ d

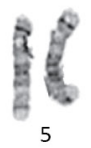

5

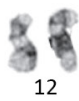

12

18

Y

Extended Data Figure 3 | Genetic stability of ES cells derived from CRISPR-Cas9-injected embryos. a, Origin and genotypes of ES cells derived from CRISPR-Cas9-injected embryos. b, Karyotype of ES-WT3 showing a pericentric inversion on chromosome 10. c, Magnified image

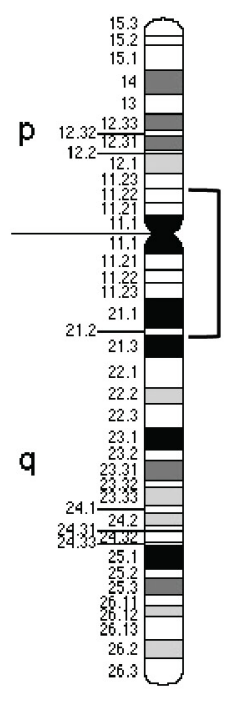

10

Normal

showing normal (left chromosome) and inverted chromosome 10 (right chromosome, inverted region indicated by arrow). d, Chromosome 10 ideogram showing the location of the inversion. 
Cas9

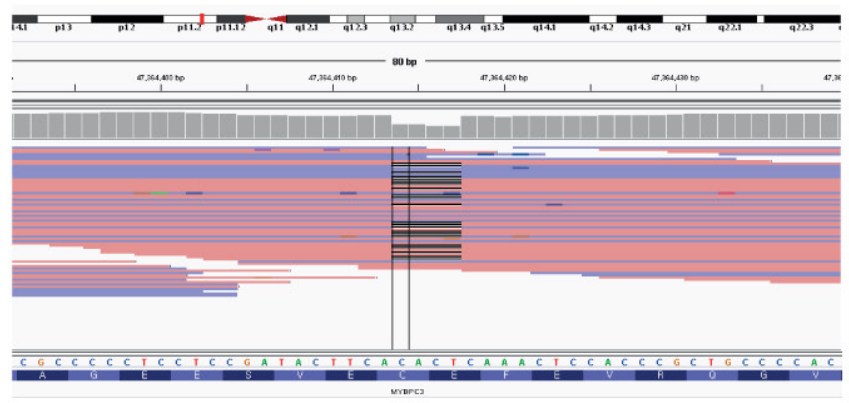

CRISPR-Cas9

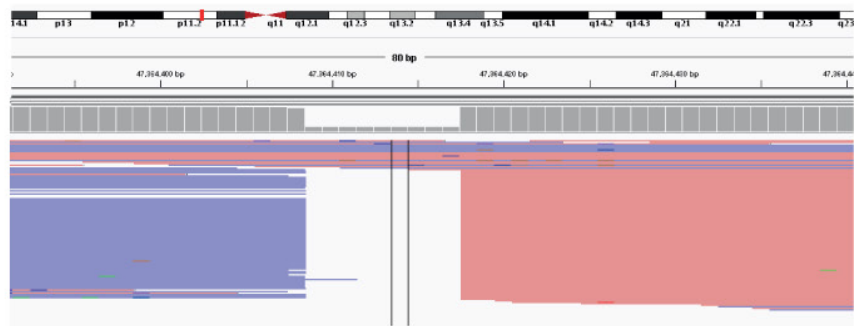

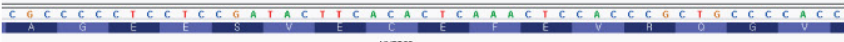

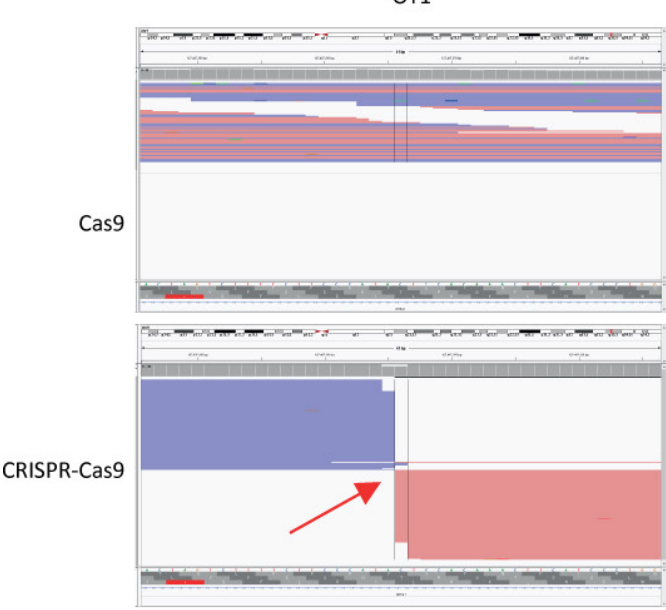

OT4

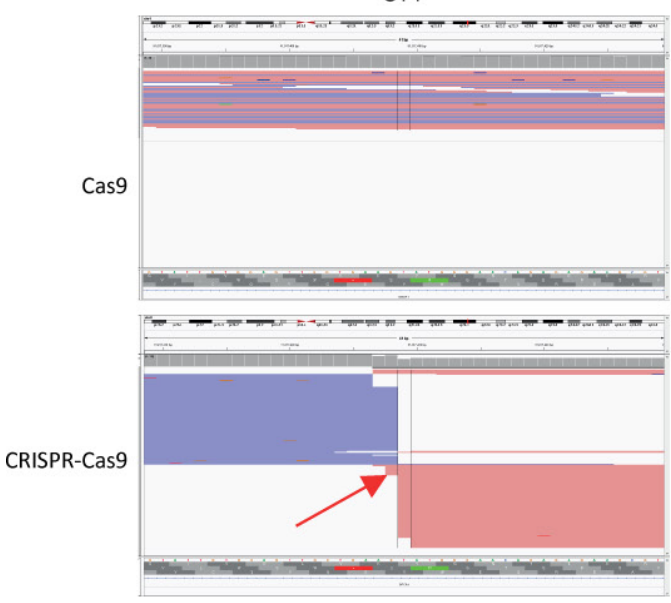

OT2

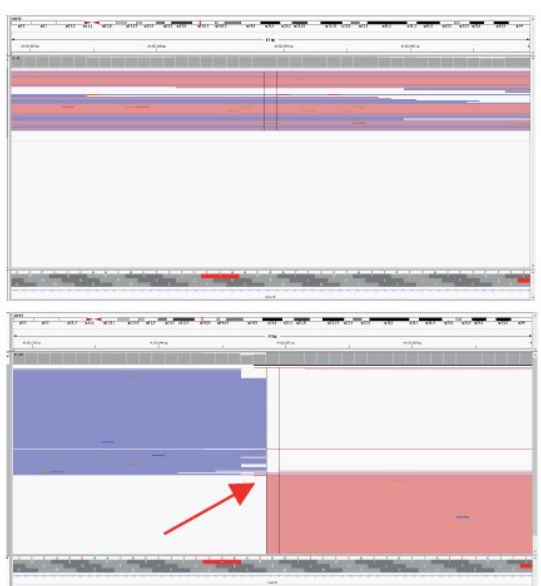

OT5
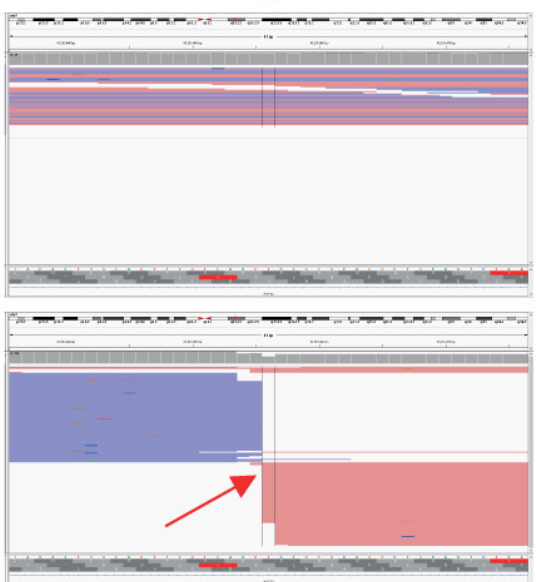

OT3

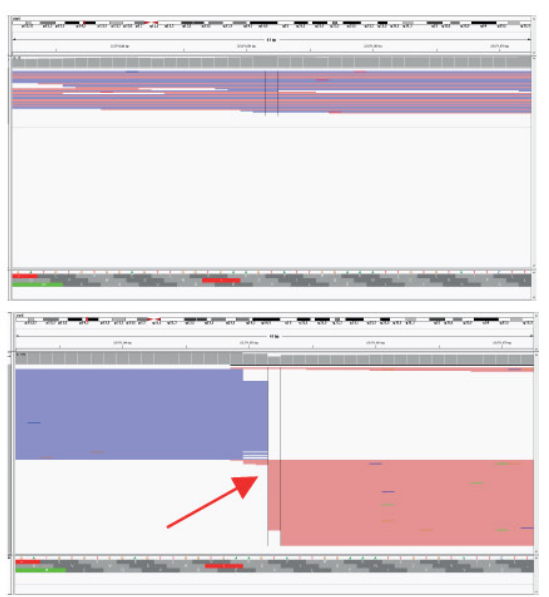

OT6

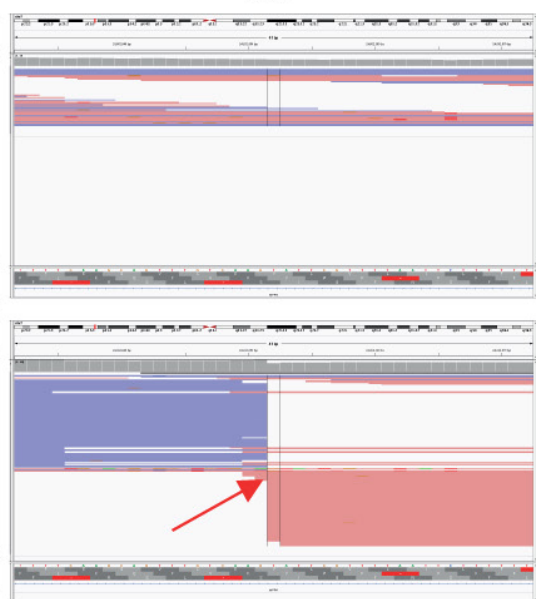

Extended Data Figure $4 \mid$ Digenome sequencing of potential off-target sites. a, Representative Integrative Genomics Viewer (IGV) images produced using CRISPR-Cas9 at the on-target site. b, Representative IGV images showing CRISPR-Cas9-induced DNA cleavage at the potential off-target sites. Red arrows indicate DNA cleavage sites at each off-target site. 
a

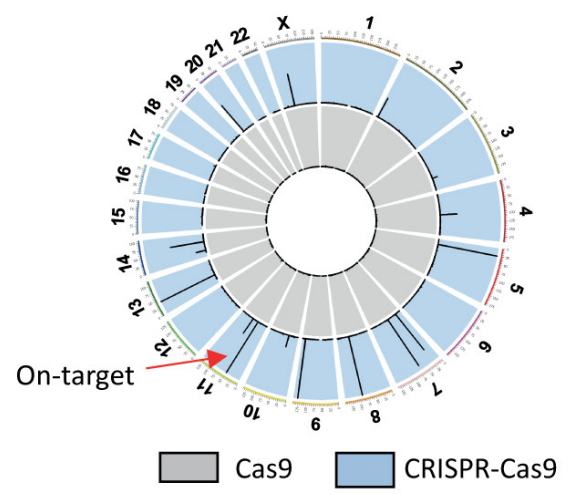

d

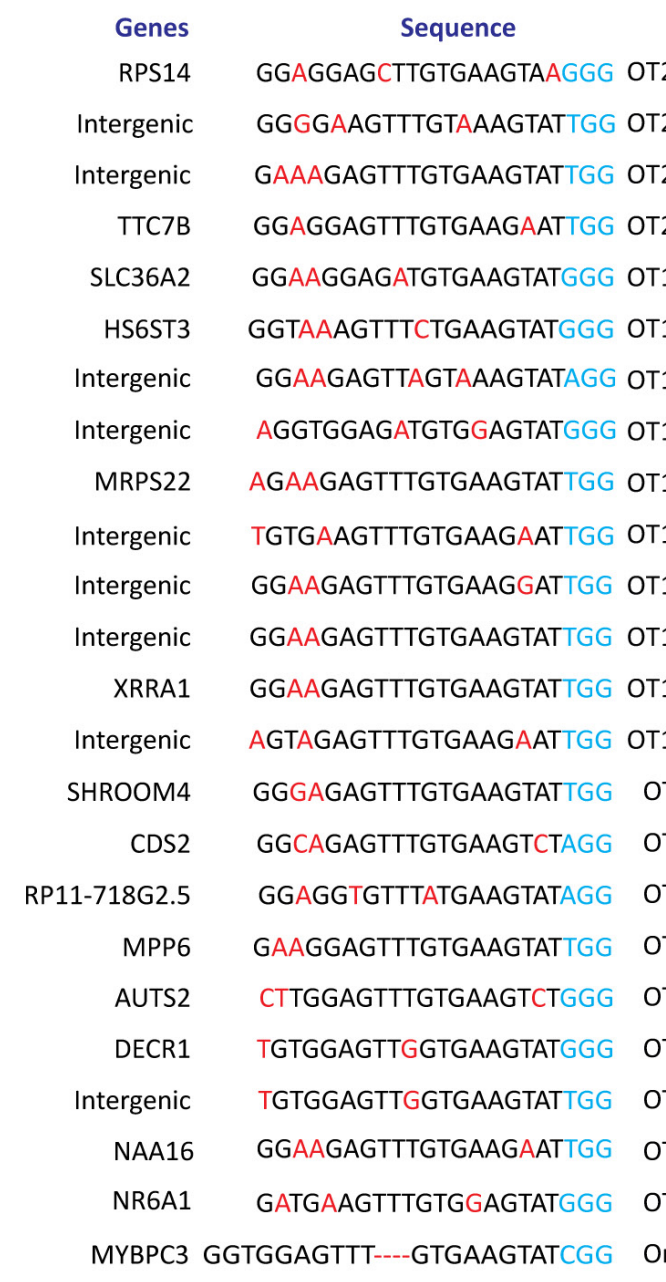

Extended Data Figure 5 | Digenome-seq based off-target mutation screening of treated human embryos. a, Genome-wide Circos plots showing DNA cleavage scores. Cas9-only-treated DNA is shown in grey, and CRISPR-Cas9-treated DNA is in blue. b, Sequence logo obtained via WebLogo using Digenome-captured sites (DNA cleavage score $>2.5$ ). On-target sequence is indicated below the sequence logo. PAM sequence is shown in blue. c, On-target indels for 28 blastomeres detected by Digenome-seq. Only blastomeres carrying NHEJ were captured by b

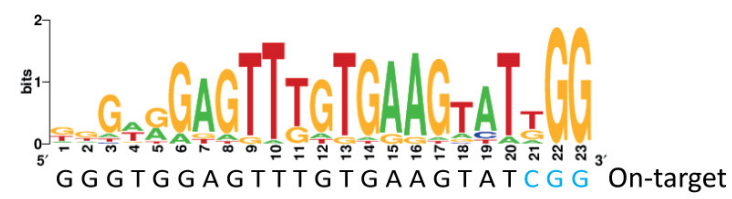

c
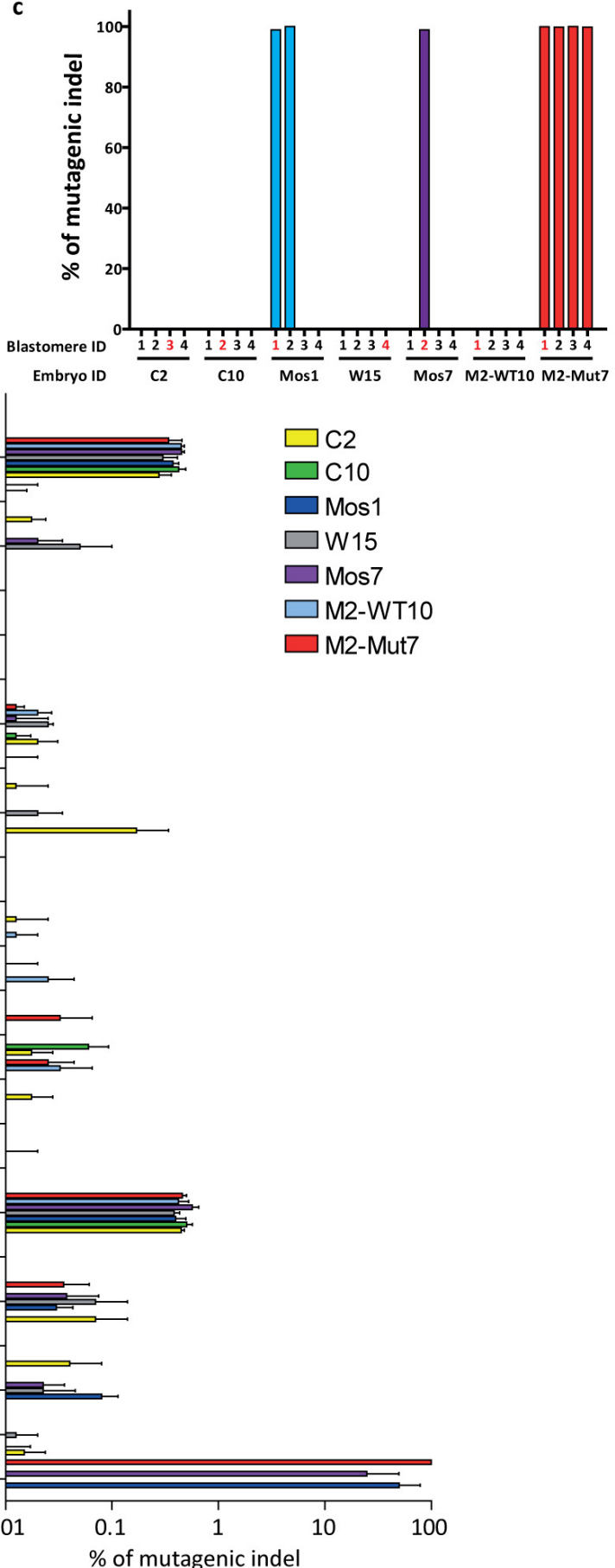

Digenome-seq. Blastomeres indicated in red fonts were also analysed by WGS (Extended Data Table 6). d, Indel frequencies for potential off-target sites captured by Digenome-seq in CRISPR-Cas9-treated $(n=5)$ and untreated control embryos $(n=2)$. Mismatch nucleotides are shown in red. PAM sequence is shown in blue. OnT: on target site; OT: off-target. Percentages of mutagenic indels on the $x$-axis are presented on a logarithmic scale. For source data, see Supplementary Table 5. 
a

Percent of variants represented in databases

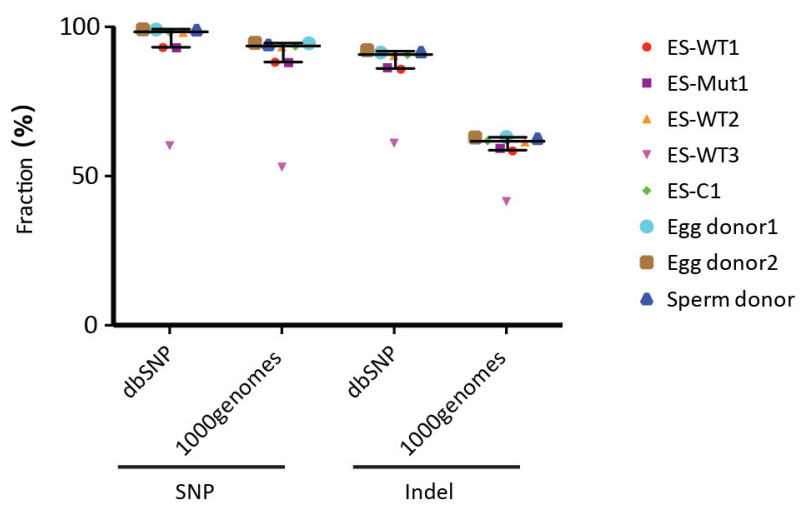

b Coding region variants-SNP (Ref.hg19)

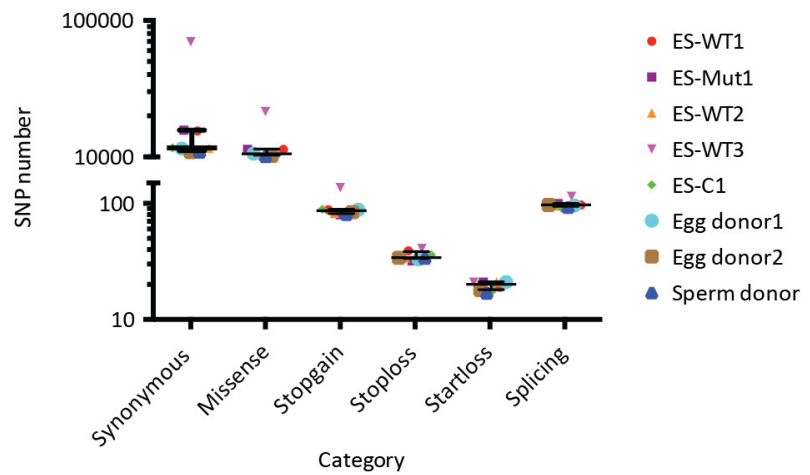

Extended Data Figure 6 | WES analysis of ES cell lines derived from CRISPR-Cas9-injected embryos. a, SNP and indel fractions of variants identified in each sample presented in dbSNP and 1000genomes databases. b, Numbers of coding region variants (SNPs) in each sample divided into c

Coding region variants-Indel (Ref.hg19)

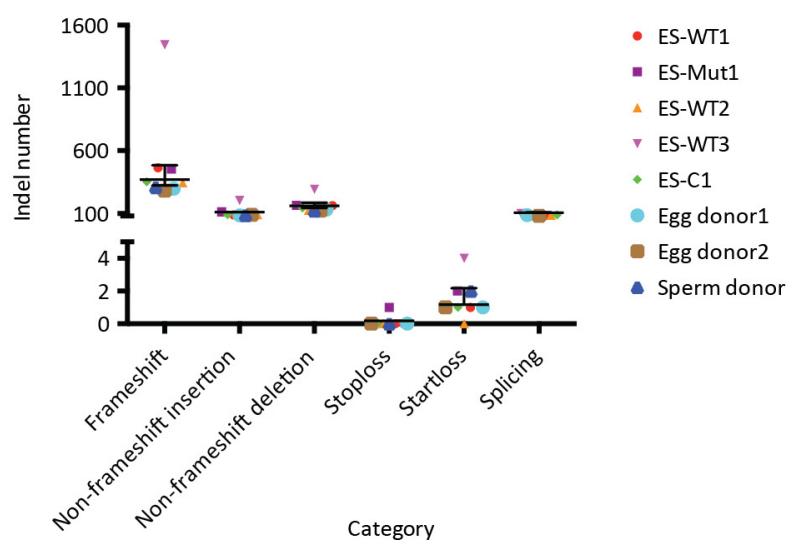

indicated categories. c, Numbers of coding region variants (indels) divided into indicated categories. $\mathrm{Hg} 19$ was used as a reference genome for variant calling. Data presented as mean with interquartile range (black horizontal bars). For source data, see Supplementary Tables 6-8. 
Extended Data Table 1 | DNA oligo sequences used for CRISPR-Cas9-based gene correction

\begin{tabular}{|c|c|c|c|}
\hline \multirow{5}{*}{ CRISPR-Cas9-1 } & \multirow{2}{*}{$\begin{array}{l}\text { Guide oligo for cloning of sgRNA (19bp)- } \\
\text { encoding plasmid }\end{array}$} & Forward & CACCGGGTGGAGTTTGTGAAGTAT \\
\hline & & Reverse & AAACATACTTCACAAACTCCACCC \\
\hline & \multirow{2}{*}{$\begin{array}{l}\text { In vitro transcription templates for encoding } \\
\text { sgRNA }\end{array}$} & Forward & $\begin{array}{l}\text { GAAATTAATACGACTCACTATAGGTGGAGTTTGTGAAGTATGTTTTAGAGCTAGAAATAGCA } \\
\text { AG }\end{array}$ \\
\hline & & Reverse & $\begin{array}{l}\text { AAAAAAGCACCGACTCGGTGCCACTTTTTCAAGTTGATAACGGACTAGCCTTATTTTAACT } \\
\text { TGCTATTTCTAGCTCTAAAAC }\end{array}$ \\
\hline & Single-stranded oligonucleotide (ssODN-1) & $200 \mathrm{mer}$ & $\begin{array}{l}\text { AGATGGCCTCAGGGGAGCCAACCCTCATGCTCACCCTGCCTGGACAGAGCCCCCTGTGC } \\
\text { TCATCAOGCGCCCCTTGGAGGACCAGCTGGTGATGGTGGGGCAGCGGGTGGAGTTTGA } \\
\text { GTGCGAGGTATOGGAGGAGGGGGGCAATCAAATGGTGAGTTCCAGAAGCACGGGGC } \\
\text { ATGGGTGTTGGGGGCATCTGCCCAG }\end{array}$ \\
\hline \multirow{3}{*}{ CRISPR-Cas9-2 } & \multirow{2}{*}{$\begin{array}{l}\text { Guide oligo for cloning of sgRNA (20bp) - } \\
\text { encoding plasmid }\end{array}$} & Forward & TGGCTTTATATATCTTGTGGAAAGGACGAAACACCGGGTGGAGTTTGTGAAGTAT \\
\hline & & Reverse & GCCTTATTTTAACTTGCTATTTCTAGCTCTAAAACATACTTCACAAACTCCACCC \\
\hline & Single-stranded oligonucleotide (ssODN-2) & $100 \mathrm{mer}$ & $\begin{array}{l}\text { CTTCTGGAACTCACCATTTGACTTGCGCCCCCTCCTCCGATACTTCACATTCGAACTCCA } \\
\text { CCCGCTGCCCCACCATCACCAGCTGGTCCTCCAAGGGGCG }\end{array}$ \\
\hline
\end{tabular}

Note: Bold letters in ssODN-1 represent synonymous nucleotide substitutions. Underlined and bold letters in ssODN-2 represent restriction enzyme (BstBI) recognition site and synonymous substitutions, respectively. 


\section{RESEARCH ARTICLE}

Extended Data Table 2 | Preimplantation development of human oocytes and zygotes after CRISPR-Cas9 injection

\begin{tabular}{lcccc}
\hline Group & No. of oocytes & $\begin{array}{c}\text { Survived after } \\
\text { CRISPR-Cas9 injection }\end{array}$ & Fertilized & 4-8-cells \\
\hline Intact control & 22 & N/A & $21 / 22(95.5 \%)$ & $19 / 22(86.4 \%)$ \\
Zygote injection & 70 & $68 / 70(97.1 \%)$ & $58 / 70(82.9 \%)$ & $54 / 70(77.1 \%)$ \\
MII injection & 75 & $73 / 75(97.3 \%)$ & $63 / 75(84.0 \%)$ & $58 / 75(77.3 \%)$ \\
\hline
\end{tabular}

N/A, not applicable. 
ARTICLE RESEARCH

Extended Data Table 3 | Genotypes of blastomeres in mosaic human embryos produced by CRISPR-Cas9 injection into zygotes

\begin{tabular}{|c|c|c|c|c|c|c|c|c|}
\hline \multirow{2}{*}{ Embryo ID } & \multirow{2}{*}{ Treatment } & \multirow{2}{*}{ No. of Blastomeres } & \multirow{2}{*}{$\begin{array}{c}\begin{array}{c}\text { Maternal } \\
\text { MYBPC3 allele }\end{array} \\
\text { Intact WT }\end{array}$} & \multicolumn{5}{|c|}{ Paternal MYBPC3 allele } \\
\hline & & & & $\begin{array}{l}\text { Intact } \\
\text { mutant }\end{array}$ & $\begin{array}{l}\text { HDR via } \\
\text { WT allele }\end{array}$ & NHEJ & Indels & No. of genotypes \\
\hline Mos1 & CRISPR-Cas9+ssODN & 8 & 8 & 0 & 6 & 2 & 5 bp deletion $(n=2)$ & 2 \\
\hline Mos2 & CRISPR-Cas9+SsODN & 3 & 3 & 0 & 2 & 1 & 1 bp insertion $(n=1)$ & 2 \\
\hline Mos3 & CRISPR-Cas9+ssODN & 6 & 6 & 0 & 1 & 5 & 3 bp deletion $(n=5)$ & 2 \\
\hline Mos4 & CRISPR-Cas9+ssODN & 4 & 4 & 0 & 3 & 1 & 1 bp insertion $(n=1)$ & 2 \\
\hline Mos5 & CRISPR-Cas9+ssODN & 7 & 7 & 3 & 2 & 2 & 12 bp deletion $(n=2)$ & 3 \\
\hline Mos6 & CRISPR-Cas9 alone & 3 & 3 & 0 & 2 & 1 & 17 bp deletion $(n-1)$ & 2 \\
\hline Mos7 & CRISPR-Cas9 alone & 4 & 4 & 0 & 3 & 1 & 5 bp deletion $(n=1)$ & 2 \\
\hline Mos8 & CRISPR-Cas9 alone & 4 & 4 & 2 & 2 & 0 & NA & 2 \\
\hline Mos9 & CRISPR-Cas9 alone & 5 & 5 & 0 & 0 & 5 & $\begin{array}{c}1 \text { bp deletion }(n=3) \\
20 \text { bp deletion }(n=1) \\
10 \text { bp deletion }+5 \text { bp } \\
\text { insertion }(n=1)\end{array}$ & 4 \\
\hline Mos10 & CRISPR-Cas9 alone & 6 & 6 & 2 & 4 & 0 & NA & 2 \\
\hline Mos11 & CRISPR-Cas9 alone & 3 & 3 & 0 & 2 & 1 & 7 bp deletion $(n=1)$ & 2 \\
\hline Mos12 & CRISPR-Cas9 alone & 8 & 8 & 0 & 7 & 1 & 1 bp insertion $(n=1)$ & 2 \\
\hline Mos13 & CRISPR-Cas9 alone & 6 & 6 & 5 & 1 & 0 & NA & 2 \\
\hline
\end{tabular}


RESEARCH ARTICLE

Extended Data Table 4 | List of potential off-target sites captured by Digenome-seq

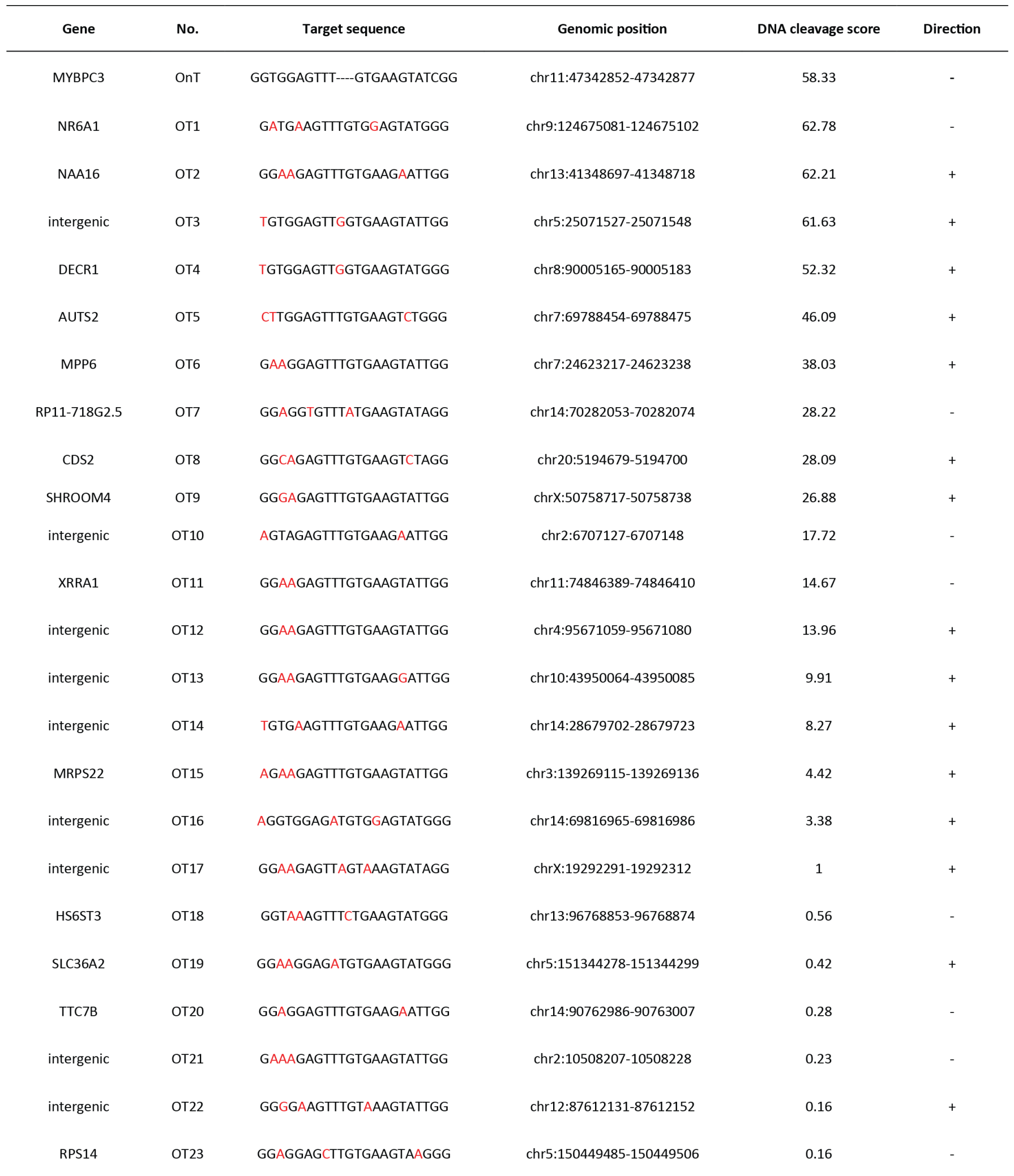

OnT, on-target; OT, off-target; captured by Digenome sequencing. Red letters indicate mismatched nucleotides. 
Extended Data Table 5 | List of PCR primers used in targeted deep sequencing

\begin{tabular}{|c|c|c|c|c|}
\hline \multirow[b]{2}{*}{ No. } & \multicolumn{2}{|c|}{$1^{\text {st }}$ PCR } & \multicolumn{2}{|c|}{$2^{\text {nd }} P C R$} \\
\hline & $\begin{array}{l}\text { Forward } \\
\left(5^{\prime}->3^{\prime}\right)\end{array}$ & $\begin{array}{l}\text { Reverse } \\
\left(5^{\prime}->3^{\prime}\right)\end{array}$ & Forward (5' ->3') & Reverse (5' ->3') \\
\hline OnT & $\begin{array}{l}\text { CCCACCCAGGTACATC } \\
\text { TITG }\end{array}$ & $\begin{array}{l}\text { TGATCAGGTGGTGTCT } \\
\text { CTGC }\end{array}$ & $\begin{array}{l}\text { ACACTCTTTCCCTACACGACGCTCTTCCGATCTACCT } \\
\text { GAGGATGTGGGAACCT }\end{array}$ & $\begin{array}{l}\text { GTGACTGGAGTTCAGACGTGTGCTCTTCCGATCTCAA } \\
\text { GTGCTGTGGCCTCTTCT }\end{array}$ \\
\hline оT1 & $\begin{array}{l}\text { CAGCAACATAAGCCA } \\
\text { GTCCA }\end{array}$ & $\begin{array}{l}\text { AAGAGAGGCCCAATG } \\
\text { GAAAT }\end{array}$ & $\begin{array}{l}\text { ACACTCTTTCCCTACACGACGCTCTTCCGATCTCAG } \\
\text { CAACATAAGCCAGTCCA }\end{array}$ & $\begin{array}{l}\text { GTGACTGGAGTTCAGACGTGTGCTCTTCCGATCTAG } \\
\text { AAATGCCTTCCTGCCTTA }\end{array}$ \\
\hline от2 & $\begin{array}{l}\text { CAGGGTGATACTGGC } \\
\text { CTCAT }\end{array}$ & $\begin{array}{l}\text { AACCCAAAACCTGGCT } \\
\text { CTIT }\end{array}$ & $\begin{array}{l}\text { ACACTCTTTCCCTACACGACGCTCTTCCGATCTCAG } \\
\text { GGTGATACTGGCCTCAT }\end{array}$ & $\begin{array}{l}\text { GTGACTGGAGTTCAGACGTGTGCTCTTCCGATCTGTC } \\
\text { CAGGCCCATATGACTTC }\end{array}$ \\
\hline OT3 & $\begin{array}{l}\text { TGTGAGGCAGAATCC } \\
\text { TTTGA }\end{array}$ & $\begin{array}{l}\text { CACAGGCATATGAAAC } \\
\text { ACTGG }\end{array}$ & $\begin{array}{l}\text { ACACTCTTTCCCTACACGACGCTCTTCCGATCTTCA } \\
\text { AAGTGTGAGGCAGAATCC }\end{array}$ & $\begin{array}{l}\text { GTGACTGGAGTTCAGACGTGTGCTCTTCCGATCTCCC } \\
\text { TCTATCAAGCTGCTATCTCA }\end{array}$ \\
\hline OT4 & $\begin{array}{l}\text { AAAGCTTCATGGAGG } \\
\text { AGGTG }\end{array}$ & $\begin{array}{l}\text { GATCCCATCCAAAAGC } \\
\text { AAGA }\end{array}$ & $\begin{array}{l}\text { ACACTCTTTCCCTACACGACGCTCTTCCGATCTGGA } \\
\text { ATTGTGTGGTCCCAAT }\end{array}$ & $\begin{array}{l}\text { GTGACTGGAGTTCAGACGTGTGCTCTTCCGATCTAA } \\
\text { GAAAAGCCCACCATACCC }\end{array}$ \\
\hline OT5 & $\begin{array}{l}\text { CCCTATGAAAGAGGG } \\
\text { TGCAA }\end{array}$ & $\begin{array}{l}\text { GCTGAACCGGACAGCT } \\
\text { AATC }\end{array}$ & $\begin{array}{l}\text { ACACTCTTTCCCTACACGACGCTCTTCCGATCTATA } \\
\text { GAGGCTTCAGGGGAAGG }\end{array}$ & $\begin{array}{l}\text { GTGACTGGAGTTCAGACGTGTGCTCTTCCGATCTACC } \\
\text { AAGTGCCCTTCAGGTTA }\end{array}$ \\
\hline от6 & $\begin{array}{l}\text { CACTCGTTCATTCCTG } \\
\text { GGATA }\end{array}$ & $\begin{array}{l}\text { ACAACCTTGCACTTGCT } \\
\text { CTG }\end{array}$ & $\begin{array}{l}\text { ACACTCTTTCCCTACACGACGCTCTTCCGATCTTITT } \\
\text { TGTGTGTGATGTTGTTGG }\end{array}$ & $\begin{array}{l}\text { GTGACTGGAGTTCAGACGTGTGCTCTTCCGATCTACC } \\
\text { AGCCTTCACTGCTGAAT }\end{array}$ \\
\hline OT7 & $\begin{array}{l}\text { GCAGACATCTACGGC } \\
\text { AGACA }\end{array}$ & $\begin{array}{l}\text { TGTGAGTGAGGCACCA } \\
\text { GAAG }\end{array}$ & $\begin{array}{l}\text { ACACTCTTTCCCTACACGACGCTCTTCCGATCTCAG } \\
\text { CTGGGGCTTAACAAGAG }\end{array}$ & $\begin{array}{l}\text { GTGACTGGAGTTCAGACGTGTGCTCTTCCGATCTCAC } \\
\text { CCCAACTTGTTCATTCA }\end{array}$ \\
\hline от8 & $\begin{array}{l}\text { CTTCCCTTTCAGGTTG } \\
\text { ACCA }\end{array}$ & $\begin{array}{l}\text { GCTCTCGGCCTCAGTC } \\
\text { ATAC }\end{array}$ & $\begin{array}{l}\text { ACACTCTTTCCCTACACGACGCTCTTCCGATCTTTTC } \\
\text { CCAACGCACTTTGAGT }\end{array}$ & $\begin{array}{l}\text { GTGACTGGAGTTCAGACGTGTGCTCTTCCGATCTGC } \\
\text { ATTGATGGGAAAAGGAA }\end{array}$ \\
\hline OT9 & $\begin{array}{l}\text { CAGCCTTGCATTCCTA } \\
\text { AGGT }\end{array}$ & $\begin{array}{l}\text { TCAAGAATTGAGGAGG } \\
\text { GAACA }\end{array}$ & $\begin{array}{l}\text { ACACTCTTTCCCTACACGACGCTCTTCCGATCTTCCA } \\
\text { CTTGGTCATGGCGTAT }\end{array}$ & $\begin{array}{l}\text { GTGACTGGAGTTCAGACGTGTGCTCTTCCGATCTAG } \\
\text { CCCAGGTCCAAAGAGTTा }\end{array}$ \\
\hline от10 & $\begin{array}{l}\text { TGCCTTATTTCTGCTCT } \\
\text { CAGG }\end{array}$ & $\begin{array}{l}\text { CAGATGGCTTCAGCAC } \\
\text { TTCA }\end{array}$ & $\begin{array}{l}\text { ACACTCTTTCCCTACACGACGCTCTTCCGATCTCTTC } \\
\text { CCTTGGTCATGGTGTT }\end{array}$ & $\begin{array}{l}\text { GTGACTGGAGTTCAGACGTGTGCTCTTCCGATCTCA } \\
\text { GATGGCTTCAGCACTTCA }\end{array}$ \\
\hline от11 & $\begin{array}{l}\text { CCAGATGTTAAACCAA } \\
\text { CCTTTCA }\end{array}$ & $\begin{array}{l}\text { AGCCTGGGCAACATAG } \\
\text { TGAG }\end{array}$ & $\begin{array}{l}\text { ACGACGCTCTTCCGATCTTTTT } \\
\text { TCTITC }\end{array}$ & $\begin{array}{l}\text { CGTGTGCTCTTCCGATCTCAA } \\
\text { CA }\end{array}$ \\
\hline OT12 & $\begin{array}{l}\text { ATCCCAGTTGGTCATG } \\
\text { GTGT }\end{array}$ & $\begin{array}{l}\text { TGCCAACAATTAGACA } \\
\text { AAAACA }\end{array}$ & $\begin{array}{l}\text { ACACTCTTTCCCTACACGACGCTCTTCCGATCTTTGT } \\
\text { TTCTGGATTTGGTTTCC }\end{array}$ & $\begin{array}{l}\text { GTGACTGGAGTTCAGACGTGTGCTCTTCCGATCTTCC } \\
\text { CAGGCCCAGATAGATAG }\end{array}$ \\
\hline оT13 & $\begin{array}{l}\text { TCGGAATGCACATCTC } \\
\text { TGTC }\end{array}$ & $\begin{array}{l}\text { GCTCAGATAGCCTCAA } \\
\text { AGGTG }\end{array}$ & $\begin{array}{l}\text { ACACTCTTTCCCTACACGACGCTCTTCCGATCTTTTT } \\
\text { CTGGATTTGGTTTGCT }\end{array}$ & $\begin{array}{l}\text { GTGACTGGAGTTCAGACGTGTGCTCTTCCGATCTGCT } \\
\text { CAGATAGCCTCAAAGGTG }\end{array}$ \\
\hline OT14 & $\begin{array}{l}\text { AAAGGGGGAAAAGG } \\
\text { TGAAAA }\end{array}$ & $\begin{array}{l}\text { CCAGGTCCAGATGCCT } \\
\text { TACT }\end{array}$ & $\begin{array}{l}\text { ACACTCTTTCCCTACACGACGCTCTTCCGATCTTGTT } \\
\text { GAGAGATTITGGTCTGTGA }\end{array}$ & $\begin{array}{l}\text { GTGACTGGAGTTCAGACGTGTGCTCTTCCGATCTCCA } \\
\text { GGTCCAGATGCCTTACT }\end{array}$ \\
\hline OT15 & $\begin{array}{l}\text { CTGGCCTTCCTGGGAT } \\
\text { AAAT }\end{array}$ & $\begin{array}{l}\text { AGGTGAAATGGACAAA } \\
\text { TTCCT }\end{array}$ & $\begin{array}{l}\text { ACACTCTTTCCCTACACGACGCTCTTCCGATCTCAG } \\
\text { GGTAATTTGGCCTCAG }\end{array}$ & $\begin{array}{l}\text { GTGACTGGAGTTCAGACGTGTGCTCTTCCGATCTAAC } \\
\text { TाCCCACAAGGAAATGC }\end{array}$ \\
\hline OT16 & $\begin{array}{l}\text { GCAAGAAAGGCTTGG } \\
\text { ATCTG }\end{array}$ & $\begin{array}{l}\text { GTCCTCCAGGTGGTTTT } \\
\text { TCA }\end{array}$ & $\begin{array}{l}\text { ACACTCTTTCCCTACACGACGCTCTTCCGATCTCAG } \\
\text { GGGTAAAGGAGGGAGAG }\end{array}$ & $\begin{array}{l}\text { GTGACTGGAGTTCAGACGTGTGCTCTTCCGATCTACC } \\
\text { CAAACGCTGATGACTTC }\end{array}$ \\
\hline OT17 & $\begin{array}{l}\text { GCAAGAGCTAGCTGC } \\
\text { ACGAT }\end{array}$ & $\begin{array}{l}\text { TTGGACAGGCTGGAAA } \\
\text { TAGG }\end{array}$ & $\begin{array}{l}\text { ACACTCTTTCCCTACACGACGCTCTTCCGATCTGGC } \\
\text { ACAAAAGAGGCTATGCT }\end{array}$ & $\begin{array}{l}\text { GTGACTGGAGTTCAGACGTGTGCTCTTCCGATCTAG } \\
\text { GCAGAAGCCAGAACAGTC }\end{array}$ \\
\hline OT18 & $\begin{array}{l}\text { TGCCCTGTTGAAATGA } \\
\text { ACCT }\end{array}$ & $\begin{array}{l}\text { TTCCTTCCTGCCACGTA } \\
\text { AAC }\end{array}$ & $\begin{array}{l}\text { ACACTCTTTCCCTACACGACGCTCTTCCGATCTCTG } \\
\text { GAACAATGATCGGGTCT }\end{array}$ & $\begin{array}{l}\text { GTGACTGGAGTTCAGACGTGTGCTCTTCCGATCTGC } \\
\text { CGTTTAGTCTGCTTGCTT }\end{array}$ \\
\hline от19 & $\begin{array}{l}\text { AACCGCTAGAGCCTCT } \\
\text { CCTC }\end{array}$ & $\begin{array}{l}\text { AGGGGTGAAGAACAG } \\
\text { GGACT }\end{array}$ & $\begin{array}{l}\text { ACACTCTTTCCCTACACGACGCTCTTCCGATCTCTTC } \\
\text { ACAGCGAGGGGTAGTC }\end{array}$ & $\begin{array}{l}\text { GTGACTGGAGTTCAGACGTGTGCTCTTCCGATCTTG } \\
\text { GGATGGTTGGTAACCCTA }\end{array}$ \\
\hline от20 & $\begin{array}{l}\text { AAACCAACCTTGCATT } \\
\text { CCTG }\end{array}$ & $\begin{array}{l}\text { CGCCTGAAACTGGGAA } \\
\text { ATAA }\end{array}$ & $\begin{array}{l}\text { ACACTCTTTCCCTACACGACGCTCTTCCGATCTTGTT } \\
\text { CCCTCCTITTCTGCTT }\end{array}$ & $\begin{array}{l}\text { GTGACTGGAGTTCAGACGTGTGCTCTTCCGATCTGC } \\
\text { CATGACCAAGAGGCTAAG }\end{array}$ \\
\hline ОT21 & $\begin{array}{l}\text { TCCTGTGTCTGTTGGG } \\
\text { ATGA }\end{array}$ & $\begin{array}{l}\text { GAGACAGGGTTTTGCC } \\
\text { ATGT }\end{array}$ & $\begin{array}{l}\text { ACACTCTTTCCCTACACGACGCTCTTCCGATCTTGCT } \\
\text { AGCATTITGTGGAGGA }\end{array}$ & $\begin{array}{l}\text { GTGACTGGAGTTCAGACGTGTGCTCTTCCGATCTAG } \\
\text { GCCCAGATAGCTTCACTG }\end{array}$ \\
\hline от22 & $\begin{array}{l}\text { TTGCATGAGTGCCATG } \\
\text { ACTT }\end{array}$ & $\begin{array}{l}\text { CCTTCTCCGATATCAGG } \\
\text { TTTG }\end{array}$ & $\begin{array}{l}\text { ACACTCTTTCCCTACACGACGCTCTTCCGATCTAGA } \\
\text { ATGCACAATCCACATGA }\end{array}$ & $\begin{array}{l}\text { GTGACTGGAGTTCAGACGTGTGCTCTTCCGATCTTG } \\
\text { AATCTGAATCCCTGCAT }\end{array}$ \\
\hline ОТ23 & $\begin{array}{l}\text { СCСТCССАСТСТСТСТT } \\
\text { TCC }\end{array}$ & $\begin{array}{l}\text { TCCCTGCTTTCCTTCTTT } \\
\text { GA }\end{array}$ & $\begin{array}{l}\text { ACACTCTTTCCCTACACGACGCTCTTCC } \\
\text { CTCGGGATTGAGTTCGT }\end{array}$ & $\begin{array}{l}\text { GTGACTGGAGTTCAGACGTGTGCTCTTCCGATCTCTA } \\
\text { CGCTGTGCAGCTCAAAC }\end{array}$ \\
\hline
\end{tabular}


Extended Data Table 6 | Analysis of off-target effects in CRISPR-Cas9-injected human embryos by WGS

Human embryo blastomere

Analysis

\begin{tabular}{ccccccc}
\hline C2.3 & C10.2 & Mos1.1 & W15.4 & Mos7.2 & M2-WT10.1 & M2-Mut7.1 \\
\hline $\begin{array}{c}\text { Control WT } \\
\text { embryo }\end{array}$ & $\begin{array}{c}\text { Control } \\
\text { mutant } \\
\text { embryo }\end{array}$ & $\begin{array}{c}\text { Zygote injected } \\
\text { with ssODN }\end{array}$ & $\begin{array}{c}\text { Zygote injected } \\
\text { without ssODN }\end{array}$ & $\begin{array}{c}\text { Zygote injected } \\
\text { without ssODN }\end{array}$ & $\begin{array}{c}\text { MII injected } \\
\text { with ssODN }\end{array}$ & $\begin{array}{c}\text { MII injected without ssODN } \\
\text { winO }\end{array}$ \\
\hline 1604350 & 2348616 & 3188787 & 2024463 & 2506788 & 2730525 & 1935404
\end{tabular}

\begin{tabular}{|c|c|c|c|c|c|c|c|c|}
\hline Lane1 & No. of total variants & 1604350 & 2348616 & 3188787 & 2024463 & 2506788 & 2730525 & 1935404 \\
\hline Lane2 & $\begin{array}{l}\text { No. of indels after } \\
\text { excluding dbSNPs }\end{array}$ & 90481 & 91073 & $\begin{array}{l}\text { - - - } \\
81037\end{array}$ & 51146 & 43827 & 41016 & $\begin{array}{l}--\boldsymbol{-} \\
31267\end{array}$ \\
\hline
\end{tabular}

PAM-containing homologous sites with up to 5 nucleotide mismatches + a DNA bulge of up to 2 nucleotides (465004)

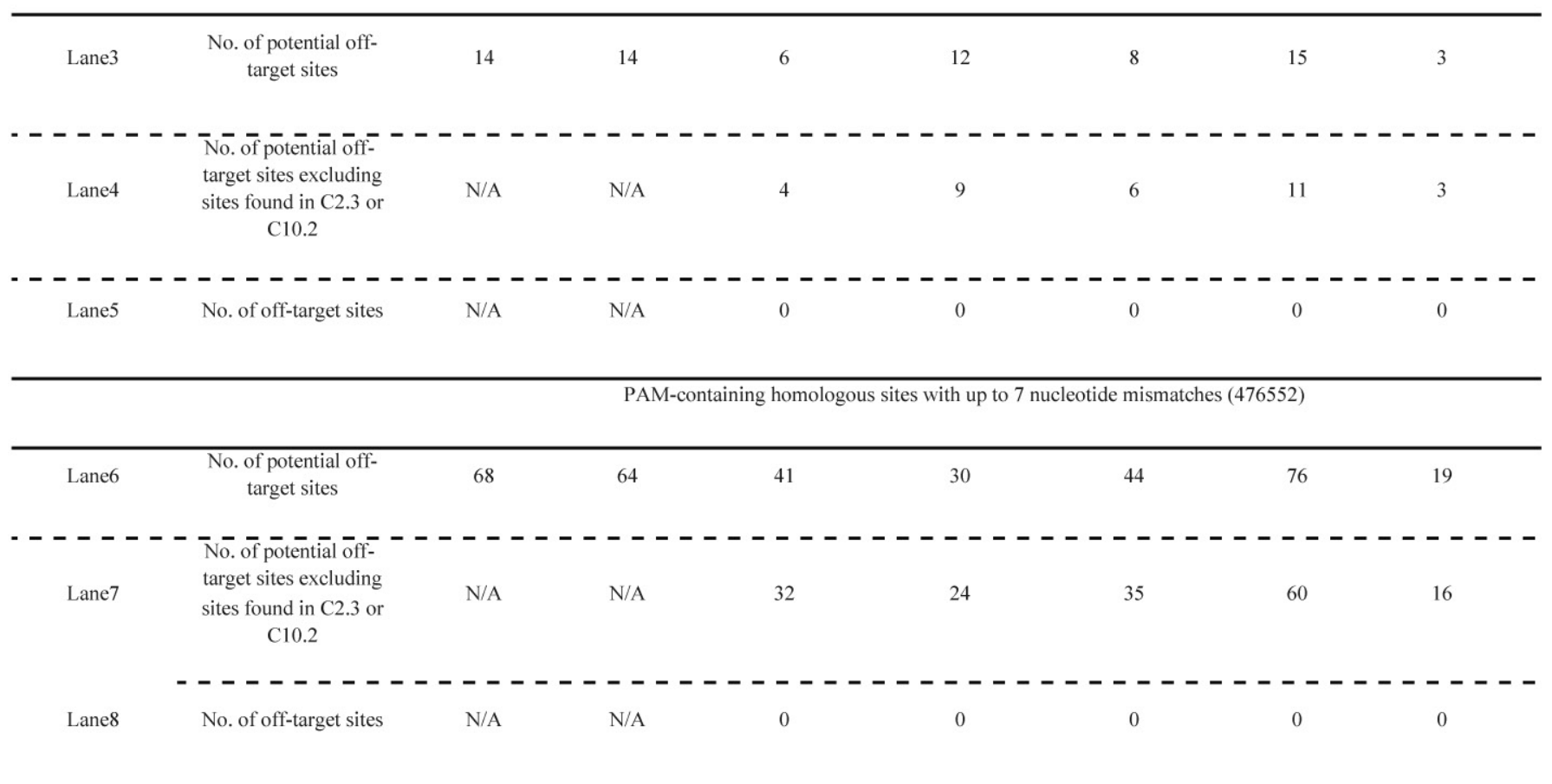

Lane 1: total number of variants including SNPs and small indels. Lane 2: number of indel sites in the human embryo blastomeres excluding dbSNPs. Lanes 3 and 6: number of potential off-target

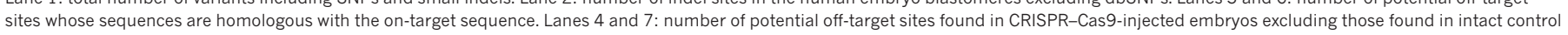
embryos (C2.3 or C10.2). Lanes 5 and 8: number of off-target sites excluding repeated sequences such as poly-A repeats or poly-GT repeats. N/A, not applicable. 


\section{Life Sciences Reporting Summary}

Nature Research wishes to improve the reproducibility of the work we publish. This form is published with all life science papers and is intended to promote consistency and transparency in reporting. All life sciences submissions use this form; while some list items might not apply to an individual manuscript, all fields must be completed for clarity.

For further information on the points included in this form, see Reporting Life Sciences Research. For further information on Nature Research policies, including our data availability policy, see Authors \& Referees and the Editorial Policy Checklist.

\section{- Experimental design}

\section{Sample size}

Describe how sample size was determined.

We used a minimum number of human oocytes/embryos required for statistical comparisons

2. Data exclusions

Describe any data exclusions.

no data were excluded

3. Replication

Describe whether the experimental findings were reliably reproduced.

Human embryo HDR and mosaicism findings were reproduced with multiple oocyte donations from different donors. All genotyping by Sanger were validated by deep sequencing (MiSeq) independently but two different teams.

\section{Randomization}

Describe how samples/organisms/participants were allocated into experimental groups.

Mutant and WT sperm from the heterozygous carrier was randomly picked up and injected into oocytes. CRISPR/Cas9 injection into zygotes or MII oocytes was randomized with controls

\section{Blinding}

Describe whether the investigators were blinded to group allocation during data collection and/or analysis.

During all on-target and off-target sequencing, the personnel was blinded regarding the sample origin

Note: all studies involving animals and/or human research participants must disclose whether blinding and randomization were used.

\section{Statistical parameters}

For all figures and tables that use statistical methods, confirm that the following items are present in relevant figure legends (or the Methods section if additional space is needed).

n/a $\mid$ Confirmed

X The exact sample size $(n)$ for each experimental group/condition, given as a discrete number and unit of measurement (animals, litters, cultures, etc.) A description of how samples were collected, noting whether measurements were taken from distinct samples or whether the same sample was measured repeatedly.

\A statement indicating how many times each experiment was replicated

The statistical test(s) used and whether they are one- or two-sided (note: only common tests should be described solely by name; more complex techniques should be described in the Methods section)

A description of any assumptions or corrections, such as an adjustment for multiple comparisons

The test results (e.g. $p$ values) given as exact values whenever possible and with confidence intervals noted

A summary of the descriptive statistics, including central tendency (e.g. median, mean) and variation (e.g. standard deviation, interquartile range)

Clearly defined error bars

See the web collection on statistics for biologists for further resources and guidance. 
Policy information about availability of computer code

\section{Software}

Describe the software used to analyze the data in this study.

We described all software used for sequence analyses in the method section.

For all studies, we encourage code deposition in a community repository (e.g. GitHub). Authors must make computer code available to editors and reviewers upon request. The Nature Methods guidance for providing algorithms and software for publication may be useful for any submission.

\section{- Materials and reagents}

Policy information about availability of materials

8. Materials availability

Indicate whether there are restrictions on availability of unique materials or if these materials are only available for distribution by a for-profit company.

CRISPR/Cas9, ESCs and iPSCs from this study are available for distribution following MTA

9. Antibodies

Describe the antibodies used and how they were validated for use in the system under study (i.e. assay and species).

10. Eukaryotic cell lines

a. State the source of each eukaryotic cell line used.

b. Describe the method of cell line authentication used.

c. Report whether the cell lines were tested for mycoplasma contamination.

d. If any of the cell lines used in the paper are listed in the database of commonly misidentified cell lines maintained by ICLAC, provide a scientific rationale for their use.

\section{- Animals and human research participants}

Policy information about studies involving animals; when reporting animal research, follow the ARRIVE guidelines

\section{Description of research animals}

Provide details on animals and/or animal-derived materials used in the study.

Policy information about studies involving human research participants

\section{Description of human research participants}

Describe the covariate-relevant population characteristics of the human research participants.
N/A

All ESC and iPSC lines included in the study were generated in this study

WGS, WES and Sanger sequencing, karyotyping

Yes, all cell lines were tested for mycoplasma contamination and were negative

None

N/A

Provided in detail in the material and method section 